\title{
New Approaches for the Synthesis of Heterocyclic Compounds Derived from Cyclohexan-1,3-dione with Anti-proliferative Activities
}

\author{
Rafat Milad Mohareb, ${ }^{1}$ Yara Raafat Milad ${ }^{2,3}$ and Ayat Ali Masoud ${ }^{1}$ \\ ${ }^{1}$ Department of Chemistry, Faculty of Science, Cairo University, A. R. Egypt \\ ${ }^{2}$ Jean Coutu, 531 Jarry Est, Montréal, Québec, Canada \\ ${ }^{3}$ Department of Pharmaceutical Chemistry, Faculty of Pharmacy, Cairo University, Cairo, A. R. Egypt \\ *Corresponding author: E-mail: raafat_mohareb@yahoo.com
}

Received: 06-11-2020

\begin{abstract}
In the present work a series of heterocyclization reactions were adopted using cyclohexan-1,3-dione through its reaction with either furan-2-carbaldehyde or thiophene-2-carbaldehyde to give the corresponding ylidene derivatives 3a,b. The latter compounds underwent heterocyclization reactions to give thiophene and pyran derivatives $\mathbf{5 a - d}$ and $\mathbf{6 a}-\mathbf{d}$, respectively. Moreover, compounds $\mathbf{3 a}$,b reacted with elemental sulfur and phenyl isothiocyanate to give the fused thiazole derivatives 8a,b. In addition, the reaction with either of hydrazine hydrate or phenylhydrazine has given the 4-hydrazono-4,5,6,7-tetrahydro- $2 \mathrm{H}$-indazole derivatives $\mathbf{1 0 a}-\mathbf{d}$, respectively. Similarly, the reaction of either $\mathbf{3 a}$ or $\mathbf{3 b}$ with hydroxylamine hydrochloride gave the 6,7-dihydrobenzo[c]isoxazol-4(5H)-one oxime derivatives 12a and 12b, respectively. Other fused heterocyclic compounds were produced and their structures were elucidated. Evaluation of the synthesized compounds against selected cancer cell lines was performed. The most active compounds were further evaluated against tyrosine kinases and Pim-1 kinase inhibitions.
\end{abstract}

Keyword: Cyclohexan-1,3-dione, thiophene, pyrazole, isoxazole, cytotoxicity

\section{Introduction}

Within the last few years the synthesis of heterocyclic compounds attracted the attention due to the wide spectrum of their high biological activities. In addition, many compounds were considered as good synthons for fused systems that were characterized by different pharmaceutical applications. ${ }^{1-10}$ Therefore, organic chemists have been making extensive efforts to produce heterocyclic compounds by developing new and efficient synthetic transformations. Within the field of pharmaceutical chemistry, many pyrazoles, thiophenes and thiazoles were reported with a wide spectrum of biological activities that included potent analgesic, anti-convulsant, anti-inflammatory and anti-bacterial, anti-pyretic, anti-tumor, anti-parasitic, anti-microbial, anti-histaminic (H1), anti-anexiety activities in tests in mice, anti-arrhythmic and as serotonin antagonists. ${ }^{1-23}$ The present work is dealing with the current application of pyrazole, thio- phene, pyrimidine and oxazine cores in the designing of anticancer agents within tumor progression. In our research it was possible to verify that such compounds are readily applicable to provide new insights and valuable inspiration in the research of new drugs and in their development as well as to contribute to the management of cancer. This encouraged our group to be attracted toward the synthesis of pyran derivatives research through the uses of b-diketones. The produced heterocyclic compounds showed high anti-proliferative activities against cancer cell lines together with high inhibitions toward tyrosine kinases. ${ }^{24-26}$ Based on such importance of heterocyclic compounds, therefore, we studied the reaction of cyclohexane-1,3-dione with some heterocyclic aldehydes and cyanomethylene reagents followed by heterocyclization of the products. Additionally, the anti-tumor evaluations of the resulting compounds towards cancer cell lines are reported. 


\section{Experimental}

\section{1. General}

The melting points of the obtained compounds were determined using Electrothermal digital melting point apparatus and are uncorrected. IR spectra ( $\mathrm{KBr}$ discs) were measured on an FTIR plus 460 or PyeUnicam SP1000 spectrophotometer (PyeUnicam, UK, Cambridge). ${ }^{1} \mathrm{H}$ NMR spectra were obtained using Varian Gemini-300 (300 MHz, Varian UK) using DMSO- $d_{6}$ as the solvent and tetramethylsilane (TMS) as the internal standard; chemical shifts are expressed as $\delta$ ppm. The mass spectra were measured with Hewlett Packard 5988 A GC/MS system (Hewlett-Packard, Agilent, USA) instrument.

\section{1. 1. General Procedure for the Synthesis of the 2-Methylenecyclohexane-1,3-dione Derivatives 3a,b}

Either of furan-2-carbaldehyde $(0.96 \mathrm{~g}, 0.01 \mathrm{~mol})$ of thiophene-2-carbaldehyde ( $1.12 \mathrm{~g}, 0.01 \mathrm{~mol})$ was added to a solution of cyclohexane-1,3-dione (1) $(1.12 \mathrm{~g}, 0.01 \mathrm{~mol})$ in absolute ethanol $(40 \mathrm{~mL})$ containing piperdine $(0.50$ $\mathrm{mL}$ ). The reaction mixture, in each case, was heated under reflux for $3 \mathrm{~h}$ then poured onto ice/water containing a few drops of hydrochloric acid and the formed solid product was collected by filtration.

\section{2-(Furan-2-ylmethylene)cyclohexane-1,3-dione (3a)}

Yellow crystals from ethanol, yield $1.46 \mathrm{~g}$ (77\%), m.p. $185-188^{\circ} \mathrm{C}$. IR $(\mathrm{KBr}) v_{\max }\left(\mathrm{cm}^{-1}\right) 3053$ (CH, aromatic), 1704, 1687 (2 CO), $1632(\mathrm{C}=\mathrm{C}) ;{ }^{1} \mathrm{H}$ NMR (DMSO- $d_{6}$, $300 \mathrm{MHz}) \delta 1.45-1.69\left(\mathrm{~m}, 2 \mathrm{H}, \mathrm{CH}_{2}\right), 2.63-2.76(\mathrm{~m}, 4 \mathrm{H}$, $\left.2 \mathrm{CH}_{2}\right), 6.82(\mathrm{~s}, 1 \mathrm{H}, \mathrm{CH}), 6.80-7.83(\mathrm{~m}, 3 \mathrm{H}$, furan $\mathrm{H})$; ${ }^{13} \mathrm{C}$ NMR (DMSO- $\left.d_{6}, 75 \mathrm{MHz}\right) \delta 16.4,36.5,39.2\left(3 \mathrm{CH}_{2}\right)$, $112.4,158.1(\mathrm{C}=\mathrm{CH}), 135.8,140.2,142.6,146.1$ (furan $\mathrm{C}$ ), 177.1, 179.4 (2CO). Anal. Calcd for $\mathrm{C}_{11} \mathrm{H}_{10} \mathrm{O}_{3}$ : C, 69.46; $\mathrm{H}$, 5.30. Found: C, 69.31; H, 5.52. MS: $m / z 190\left(\mathrm{M}^{+}, 28 \%\right)$.

\section{2-(Thiophen-2-ylmethylene)cyclohexane-1,3-dione (3b)}

Orange crystals from ethanol, yield $1.44 \mathrm{~g}$ (70\%), m.p. 201-204 ${ }^{\circ} \mathrm{C}$. IR ( $\left.\mathrm{KBr}\right) v_{\max }\left(\mathrm{cm}^{-1}\right) 3055(\mathrm{CH}$, aromatic), 1703, 1689 (2 CO), $1630(\mathrm{C}=\mathrm{C}) ;{ }^{1} \mathrm{H}$ NMR (DMSO- $\left.d_{6}, 200 \mathrm{MHz}\right) \delta$ 1.43-1.68 (m, $\left.2 \mathrm{H}, \mathrm{CH}_{2}\right), 2.61-2.74\left(\mathrm{~m}, 4 \mathrm{H}, 2 \mathrm{CH}_{2}\right), 6.80(\mathrm{~s}, 1 \mathrm{H}$, $\mathrm{CH}), 6.82-7.88(\mathrm{~m}, 3 \mathrm{H}$, thiophene $\mathrm{H}) ;{ }^{13} \mathrm{C}$ NMR (DMSO- $d_{6}$, $75 \mathrm{MHz}) \delta 16.8,36.2,39.1\left(3 \mathrm{CH}_{2}\right), 112.6,158.8(\mathrm{C}=\mathrm{CH})$, 135.6, 140.5, 142.3, 146.2 (thiophene C), 177.6, 179.2 (2CO). Anal. Calcd for $\mathrm{C}_{11} \mathrm{H}_{10} \mathrm{O}_{2} \mathrm{~S}$ : C, 64.05; H, 4.89; S, 15.55. Found: C, 63.80; H, 4.73; 15.38. MS: $m / z 206\left(\mathrm{M}^{+}, 32 \%\right)$.

\section{1. 2. General Procedure for the Synthesis of the 6,7-Dihydrobenzo $[b]$ thiophen-5(4H)-one Derivatives 5a-d}

Either malononitrile $(0.66 \mathrm{~g}, 0.01 \mathrm{~mol})$ or ethyl cyanoacetate $(1.07 \mathrm{~g}, 0.01 \mathrm{~mol})$ was added to a solution of either compound $\mathbf{3 a}(1.90 \mathrm{~g}, 0.01 \mathrm{~mol})$ or $\mathbf{3 b}(2.06 \mathrm{~g}, 0.01$ $\mathrm{mol})$ in ethanol $(40 \mathrm{~mL})$ containing triethylamine $(0.50$ $\mathrm{mL}$ ). The reaction mixture was heated under reflux for 3 $\mathrm{h}$ then poured onto ice/water mixture containing a few drops of hydrochloric acid and the formed solid product was collected by filtration.

\section{2-Amino-4-(furan-2-ylmethylene)-5-oxo-4,5,6,7- tetrahydrobenzo $[b]$ thiophene-3-carbonitrile (5a)}

Pale yellow crystals from ethanol, yield $1.94 \mathrm{~g}(70 \%)$, m.p. $130-132^{\circ} \mathrm{C}$. IR (KBr) $v_{\max }\left(\mathrm{cm}^{-1}\right) 3482-3353\left(\mathrm{NH}_{2}\right)$, $3056(\mathrm{CH}$, aromatic), $2220(\mathrm{CN}), 1686(\mathrm{CO}), 1630(\mathrm{C}=\mathrm{C})$; ${ }^{1} \mathrm{H}$ NMR (DMSO- $\left.d_{6}, 300 \mathrm{MHz}\right) \delta 2.62-2.78(2 \mathrm{t}, 4 \mathrm{H}$, $\left.2 \mathrm{CH}_{2}\right), 4.73\left(\mathrm{~s}, 2 \mathrm{H}, \mathrm{D}_{2} \mathrm{O}\right.$ exchangeable, $\left.\mathrm{NH}_{2}\right), 6.84(\mathrm{~s}, 1 \mathrm{H}$, $\mathrm{CH}), 6.82-7.86(\mathrm{~m}, 3 \mathrm{H}$, furan $\mathrm{H}) ;{ }^{13} \mathrm{C}$ NMR (DMSO- $d_{6}$, $75 \mathrm{MHz}) \delta 16.6,36.3,39.5\left(3 \mathrm{CH}_{2}\right), 112.6,158.4(\mathrm{C}=\mathrm{CH})$, 116.8 (CN), 135.4, 140.6, 141.4, 142.2, 142.7, 144.8, 145.6, 146.5 (thiophene, furan C), 179.3 (CO). Anal. Calcd for $\mathrm{C}_{14} \mathrm{H}_{10} \mathrm{~N}_{2} \mathrm{O}_{2} \mathrm{~S}$ : C, 62.21; H, 3.73; N, 10.36; S, 11.86. Found: C, 62.36; H, 3.80; N, 10.41; S, 12.04. MS: $m / z 270\left(\mathrm{M}^{+}, 32 \%\right)$.

Ethyl 2-Amino-4-(furan-2-ylmethylene)-5-oxo-4,5,6,7tetrahydrobenzo $[b]$ thiophene-3-carboxylate $(5 b)$

Yellow crystals from ethanol, yield $2.21 \mathrm{~g}(70 \%)$, m.p.125-127 ${ }^{\circ} \mathrm{C}$. IR (KBr) $v_{\max }\left(\mathrm{cm}^{-1}\right)$ 3494-3368 $\left(\mathrm{NH}_{2}\right)$, $3058\left(\mathrm{CH}\right.$, aromatic), 2931, $2972\left(\mathrm{CH}_{2}, \mathrm{CH}_{3}\right), 1689,1688$ (2 CO), 1630 (C=C); ${ }^{1} \mathrm{H}$ NMR (DMSO- $\left.d_{6}, 300 \mathrm{MHz}\right) \delta$ $1.12\left(\mathrm{t}, 3 \mathrm{H}, J=7.28 \mathrm{~Hz}, \mathrm{CH}_{3}\right), 2.68-2.74\left(2 \mathrm{t}, 4 \mathrm{H}, 2 \mathrm{CH}_{2}\right)$, $4.21\left(\mathrm{q}, 2 \mathrm{H}, J=7.28 \mathrm{~Hz}, \mathrm{CH}_{2}\right), 4.76\left(\mathrm{~s}, 2 \mathrm{H}, \mathrm{D}_{2} \mathrm{O}\right.$ exchangeable, $\left.\mathrm{NH}_{2}\right), 6.86(\mathrm{~s}, 1 \mathrm{H}, \mathrm{CH}), 6.84-7.92(\mathrm{~m}, 3 \mathrm{H}$, furan $\mathrm{H})$; ${ }^{13} \mathrm{C} \mathrm{NMR}\left(\mathrm{DMSO}-d_{6}, 75 \mathrm{MHz}\right) \delta 16.3\left(\mathrm{OCH}_{2} \underline{\mathrm{CH}}_{3}\right), 16.4$, $36.8,39.3\left(3 \mathrm{CH}_{2}\right), 52.8\left(\mathrm{OCH}_{2} \mathrm{CH}_{3}\right), 112.3,158.6(\mathrm{C}=\mathrm{CH})$, $135.6,18.0,140.8,141.6,142.3,143.5,144.2,146.7$ (thiophene, furan C), 164.8, 178.3 (2CO). Anal. Calcd for $\mathrm{C}_{16} \mathrm{H}_{15} \mathrm{NO}_{4} \mathrm{~S}: \mathrm{C}, 60.55 ; \mathrm{H}, 4.76 ; \mathrm{N}, 4.41 ; \mathrm{S}, 10.10$. Found: C, 60.80; H, 4.83; N, 4.60; S, 10.26. MS: $m / z 317\left(\mathrm{M}^{+}, 38 \%\right)$.

\section{2-Amino-5-oxo-4-(thiophen-2-ylmethylene)-4,5,6,7- tetrahydrobenzo $[b]$ thiophene-3-carbonitrile $(5 c)$}

Orange crystals from ethanol, yield $1.94 \mathrm{~g}(68 \%)$, m.p. $202-205^{\circ} \mathrm{C}$. IR $(\mathrm{KBr}) v_{\max }\left(\mathrm{cm}^{-1}\right) 3459-3342\left(\mathrm{NH}_{2}\right)$, 3058 (CH, aromatic), $2221(\mathrm{CN}), 1688(\mathrm{CO}), 1630(\mathrm{C}=\mathrm{C})$; ${ }^{1} \mathrm{H}$ NMR (DMSO- $\left.d_{6}, 300 \mathrm{MHz}\right) \delta 2.64-2.75(2 \mathrm{t}, 4 \mathrm{H}$, $\left.2 \mathrm{CH}_{2}\right), 4.74\left(\mathrm{~s}, 2 \mathrm{H}, \mathrm{D}_{2} \mathrm{O}\right.$ exchangeable, $\left.\mathrm{NH}_{2}\right), 6.88(\mathrm{~s}, 1 \mathrm{H}$, $\mathrm{CH}), 6.80-7.87(\mathrm{~m}, 3 \mathrm{H}$, thiophene $\mathrm{H}) ;{ }^{13} \mathrm{C}$ NMR (DMSO- $\left.d_{6}, 75 \mathrm{MHz}\right) \delta 16.3,39.8\left(2 \mathrm{CH}_{2}\right), 112.3,158.8(\mathrm{C}=\mathrm{CH})$, $116.9(\mathrm{CN}), 134.2,138.6,140.3,142.4,143.1,143.6,145.2$, 146.7 (two thiophene C), 179.8 (CO). Anal. Calcd for $\mathrm{C}_{14} \mathrm{H}_{10} \mathrm{~N}_{2} \mathrm{OS}_{2}$ : C, 58.72; H, 3.52; N, 9.78; S, 22.39. Found: C, 58.36; H, 3.80; N, 9.68; S, 22.41. MS: $m / z 286\left(\mathrm{M}^{+}, 46 \%\right)$.

Ethyl 2-Amino-5-oxo-4-(thiophen-2-ylmethylene)4,5,6,7-tetrahydrobenzo $[b]$ thiophene-3-carboxylate (5d)

Pale yellow crystals from ethanol, yield $2.13 \mathrm{~g}(66 \%)$, m.p. $189-192^{\circ} \mathrm{C}$. IR $(\mathrm{KBr}) v_{\max }\left(\mathrm{cm}^{-1}\right) 3486-3342\left(\mathrm{NH}_{2}\right)$, $3054\left(\mathrm{CH}\right.$, aromatic), 2938, $2893\left(\mathrm{CH}_{2}, \mathrm{CH}_{3}\right), 1689,1686$ (2 
CO), $1630\left(\mathrm{C}=\mathrm{C}\right.$ ); ${ }^{1} \mathrm{H}$ NMR (DMSO- $\left.d_{6}, 300 \mathrm{MHz}\right) \delta 1.13$ $\left(\mathrm{t}, 3 \mathrm{H}, J=7.59 \mathrm{~Hz}, \mathrm{CH}_{3}\right), 2.64-2.78\left(2 \mathrm{t}, 4 \mathrm{H}, 2 \mathrm{CH}_{2}\right), 4.23$ (q, $\left.2 \mathrm{H}, J=7.59 \mathrm{~Hz}, \mathrm{CH}_{2}\right), 4.79\left(\mathrm{~s}, 2 \mathrm{H}, \mathrm{D}_{2} \mathrm{O}\right.$ exchangeable, $\left.\mathrm{NH}_{2}\right), 6.84(\mathrm{~s}, 1 \mathrm{H}, \mathrm{CH}), 7.29-7.85(\mathrm{~m}, 3 \mathrm{H}$, thiophene $\mathrm{H})$; ${ }^{13} \mathrm{C}$ NMR (DMSO- $\left.d_{6}, 75 \mathrm{MHz}\right) \delta 16.1\left(\mathrm{OCH}_{2} \mathrm{CH}_{3}\right), 16.5$, $36.4,39.7\left(3 \mathrm{CH}_{2}\right), 52.3\left(\mathrm{OCH}_{2} \mathrm{CH}_{3}\right), 112.1,158.4(\mathrm{C}=\mathrm{CH})$, $135.3,136.7,138.3,140.5,141.5,142.0,143.8,144.6,146.8$ (two thiophene C), 164.5, 178.9 (2CO). Anal. Calcd for $\mathrm{C}_{16} \mathrm{H}_{15} \mathrm{NO}_{3} \mathrm{~S}_{2}$ : C, 57.64; H, 4.53; N, 4.20; S, 19.23. Found: C, 57.80; H, 4.71; N, 4.38; S, 19.46. MS: $m / z 333\left(\mathrm{M}^{+}, 26 \%\right)$.

\section{1. 3. General Procedure for the Synthesis of the 2H-Chromen-5-one Derivatives 6a-d}

Either malononitrile $(0.66 \mathrm{~g}, 0.01 \mathrm{~mol})$ or ethyl cyanoacetate $(1.13 \mathrm{~g}, 0.01 \mathrm{~mol})$ was added to a solution of either compound $3 \mathbf{a}(1.90 \mathrm{~g}, 0.01 \mathrm{~mol})$ or $3 \mathbf{b}(2.06 \mathrm{~g}, 0.01 \mathrm{~mol})$ in absolute ethanol $(40 \mathrm{~mL})$ containing triethylamine $(1.0 \mathrm{~mL})$. The reaction mixture, in each case, was heated under reflux for $3 \mathrm{~h}$ then the excess solvent was removed under vacuum. The remaining product was triturated with diethyl ether and the formed solid product was collected by filtration.

\section{2-Amino-4-(furan-2-yl)-5-oxo-5,6,7,8-tetrahydro- $2 \mathrm{H}$ - chromene-3-carbonitrile (6a)}

Pale yellow crystals from ethanol, yield $1.66 \mathrm{~g}(69 \%)$, m.p. $194-196^{\circ} \mathrm{C}$. IR $(\mathrm{KBr}) v_{\max }\left(\mathrm{cm}^{-1}\right) 3470-3328\left(\mathrm{NH}_{2}\right)$, $3055\left(\mathrm{CH}\right.$, aromatic), $2220(\mathrm{CN}), 1632(\mathrm{C}=\mathrm{C}) ;{ }^{1} \mathrm{H}$ NMR (DMSO- $\left.d_{6}, 300 \mathrm{MHz}\right) \delta 1.41-1.66\left(\mathrm{~m}, 4 \mathrm{H}, 2 \mathrm{CH}_{2}\right), 2.61-$ $2.76\left(\mathrm{~m}, 4 \mathrm{H}, 2 \mathrm{CH}_{2}\right), 4.71\left(\mathrm{~s}, 2 \mathrm{H}, \mathrm{D}_{2} \mathrm{O}\right.$ exchangeable, $\left.\mathrm{NH}_{2}\right)$, $6.04(\mathrm{~s}, 1 \mathrm{H}$, pyran $\mathrm{H}-4), 6.87-7.83(\mathrm{~m}, 3 \mathrm{H}$, furan $\mathrm{H}) ;{ }^{13} \mathrm{C}$ NMR (DMSO- $\left.d_{6}, 75 \mathrm{MHz}\right) \delta 16.1,36.3,37.2,39.5\left(4 \mathrm{CH}_{2}\right)$, $116.8(\mathrm{CN}), 134.3,141.5,141.8,142.6,1431.4,143.8$, 145.2, 146.4 (pyran, furan C), 178.4 (CO). Anal. Calcd for $\mathrm{C}_{14} \mathrm{H}_{14} \mathrm{~N}_{2} \mathrm{O}_{2}$ : C, 69.41; H, 5.82; N, 11.56. Found: C, 69.38; $\mathrm{H}, 5.92 ; \mathrm{N}, 11.29$. MS: $\mathrm{m} / z 242\left(\mathrm{M}^{+}, 28 \%\right)$.

\section{4-(Furan-2-yl)-2-hydroxy-5-oxo-5,6,7,8-tetrahydro-4H-} chromene-3-carbonitrile (6b)

Pale brown crystals from ethanol, yield $1.79 \mathrm{~g}(74 \%)$, m.p. $198-200^{\circ} \mathrm{C}$. IR (KBr) $v_{\max }\left(\mathrm{cm}^{-1}\right) 3568-3339(\mathrm{OH})$, $3055\left(\mathrm{CH}\right.$, aromatic), $2222(\mathrm{CN}), 1630(\mathrm{C}=\mathrm{C}) ;{ }^{1} \mathrm{H}$ NMR $\left(\right.$ DMSO- $\left.d_{6}, 300 \mathrm{MHz}\right) \delta 1.32-1.68\left(\mathrm{~m}, 4 \mathrm{H}, 2 \mathrm{CH}_{2}\right), 2.63-$ $2.74\left(\mathrm{~m}, 4 \mathrm{H}, 2 \mathrm{CH}_{2}\right), 6.02(\mathrm{~s}, 1 \mathrm{H}$, pyran $\mathrm{H}-4), 6.84-7.85(\mathrm{~m}$, $3 \mathrm{H}$, furan $\mathrm{H}), 9.80\left(\mathrm{~s}, 1 \mathrm{H}, \mathrm{D}_{2} \mathrm{O}\right.$ exchangeable, $\left.\mathrm{OH}\right) ;{ }^{13} \mathrm{C}$ NMR (DMSO- $\left.d_{6}, 75 \mathrm{MHz}\right) \delta 16.3,36.5,37.1,39.3\left(4 \mathrm{CH}_{2}\right)$, 116.7 (CN), 134.1, 138.0, 140.2, 141.7, 142.3, 142.9, 143.3, 146.6 (furan, pyran C). Anal. Calcd for $\mathrm{C}_{14} \mathrm{H}_{13} \mathrm{NO}_{3}$ : C, 69.12; H, 5.39; N, 5.76. Found: C, 68.92; H, 5.42; N, 5.58. MS: $m / z 243\left(\mathrm{M}^{+}, 36 \%\right)$.

\section{2-Amino-4-(thiophen-2-yl)-5,6,7,8-tetrahydro- $4 H$ - chromene-3-carbonitrile (6c)}

Brown crystals from ethanol, yield $1.85 \mathrm{~g}(72 \%)$, m.p. 211-214 ${ }^{\circ} \mathrm{C}$. IR $(\mathrm{KBr}) v_{\max }\left(\mathrm{cm}^{-1}\right) 3445-3372\left(\mathrm{NH}_{2}\right)$, $3055\left(\mathrm{CH}\right.$, aromatic), $2220(\mathrm{CN}), 1630(\mathrm{C}=\mathrm{C}) ;{ }^{1} \mathrm{H}$ NMR
(DMSO- $\left.d_{6}, 200 \mathrm{MHz}\right) \delta 1.41-1.64\left(\mathrm{~m}, 4 \mathrm{H}, 2 \mathrm{CH}_{2}\right), 2.61-$ $2.77\left(\mathrm{~m}, 4 \mathrm{H}, 2 \mathrm{CH}_{2}\right), 4.73\left(\mathrm{~s}, 2 \mathrm{H}, \mathrm{D}_{2} \mathrm{O}\right.$ exchangeable, $\left.\mathrm{NH}_{2}\right)$, $6.07(\mathrm{~s}, 1 \mathrm{H}$, thiophene $\mathrm{H}-4), 6.91-7.73(\mathrm{~m}, 3 \mathrm{H}$, thiophene $\mathrm{H}$ ); ${ }^{13} \mathrm{C}$ NMR (DMSO- $\left.d_{6}, 75 \mathrm{MHz}\right) \delta 16.3,36.6,37.2$, $39.8\left(4 \mathrm{CH}_{2}\right), 116.4(\mathrm{CN}), 134.6,138.7,140.2,141.6,142.3$, 142.8, 143.6, 146.2 (thiophene, pyran C). Anal. Calcd for $\mathrm{C}_{14} \mathrm{H}_{14} \mathrm{~N}_{2} \mathrm{O}_{2} \mathrm{~S}$ : C, 65.09; H, 5.46; N, 10.84; S, 12.41. Found: C, 64.90; H, 5.62; N, 10.68; S, 12.58. MS: $m / z 258\left(\mathrm{M}^{+}, 40 \%\right)$.

\section{2-Hydroxy-4-(thiophen-2-yl)-5,6,7,8-tetrahydro-4H- chromene-3-carbonitrile (6d)}

Orange crystals from ethanol, yield $1.96 \mathrm{~g}(76 \%)$, m.p. $177-179^{\circ} \mathrm{C}$. IR $(\mathrm{KBr}) v_{\max }\left(\mathrm{cm}^{-1}\right) 3542-3329(\mathrm{OH})$, $3055(\mathrm{CH}$, aromatic), $2220(\mathrm{CN}), 1688(\mathrm{CO}), 1630(\mathrm{C}=\mathrm{C})$; ${ }^{1} \mathrm{H}$ NMR (DMSO- $\left.d_{6}, 300 \mathrm{MHz}\right) \delta 1.34-1.66(\mathrm{~m}, 4 \mathrm{H}$, $\left.2 \mathrm{CH}_{2}\right), 2.61-2.76\left(\mathrm{~m}, 4 \mathrm{H}, 2 \mathrm{CH}_{2}\right), 6.04(\mathrm{~s}, 1 \mathrm{H}$, pyran $\mathrm{H}-4)$, 6.76-7.89 $(\mathrm{m}, 3 \mathrm{H}$, furan $\mathrm{H}), 9.83\left(\mathrm{~s}, 1 \mathrm{H}, \mathrm{D}_{2} \mathrm{O}\right.$ exchangeable, OH); ${ }^{13} \mathrm{C}$ NMR (DMSO- $\left.d_{6}, 75 \mathrm{MHz}\right) \delta 16.1,36.3,37.4$, $39.6\left(4 \mathrm{CH}_{2}\right), 116.9(\mathrm{CN}), 134.4,139.2,140.8,141.5,143.7$, 144.3, 145.2, 146.8 (furan, pyran C), 178.6 (CO). Anal. Calcd for $\mathrm{C}_{14} \mathrm{H}_{13} \mathrm{NO}_{2} \mathrm{~S}$ : C, 64.84; $\mathrm{H}, 5.05 ; \mathrm{N}, 5.40 ; \mathrm{S}, 12.36$. Found: $\mathrm{C}, 64.72 ; \mathrm{H}, 5.24 ; \mathrm{N}, 12.59$. MS: $\mathrm{m} / z 259\left(\mathrm{M}^{+}, 42 \%\right)$.

\section{1. 4. General Procedure for the Synthesis of 2-Thioxohexahydrobenzo $[d]$ thiazole Derivatives 8a,b}

Each of elemental sulfur $(0.32 \mathrm{~g}, 0.01 \mathrm{~mol})$ and phenyl isothiocyanate $(1.30 \mathrm{~g}, 0.01 \mathrm{~mol})$ were added to a solution of either compound $\mathbf{3 a}(1.90 \mathrm{~g}, 0.01 \mathrm{~mol})$ or $\mathbf{3 b}(2.06$ $\mathrm{g}, 0.01 \mathrm{~mol})$ in 1,4-dioxane $(40 \mathrm{~mL})$ containing triethylamine $(1.0 \mathrm{~mL})$. The reaction mixture was heated under reflux for $2 \mathrm{~h}$ then was left to cool and the formed solid product, in each case, was collected by filtration.

\section{4-(Furan-2-ylmethylene)-3-phenyl-2-thioxo-2,3,6,7- tetrahydrobenzo $[d]$ thiazol-5 $(4 \mathrm{H})$-one $(8 \mathrm{a})$}

Yellow crystals from ethanol, yield $2.50 \mathrm{~g}(74 \%)$, m.p. 168-169 ${ }^{\circ} \mathrm{C}$. IR $(\mathrm{KBr}) v_{\max }\left(\mathrm{cm}^{-1}\right) 3055(\mathrm{CH}$, aromatic), $1688(\mathrm{CO}), 1630(\mathrm{C}=\mathrm{C}), 1205(\mathrm{C}=\mathrm{S})$; ${ }^{1} \mathrm{H}$ NMR (DMSO- $d_{6}$, $300 \mathrm{MHz}) \delta 2.63-2.78\left(2 \mathrm{t}, 4 \mathrm{H}, 2 \mathrm{CH}_{2}\right), 6.87(\mathrm{~s}, 1 \mathrm{H}, \mathrm{CH})$, 6.82-7.85 (m, $8 \mathrm{H}, \mathrm{C}_{6} \mathrm{H}_{5}$, furan $\left.\mathrm{H}\right) ;{ }^{13} \mathrm{C}$ NMR (DMSO- $d_{6}$, $75 \mathrm{MHz}) \delta 16.1,36.6,39.5\left(3 \mathrm{CH}_{2}\right), 112.1,158.4(\mathrm{C}=\mathrm{CH})$, $120.3,122.6,124.8,127.2,134.6,140.8,142.2,143.2,143.8$, $146.9\left(\mathrm{C}_{6} \mathrm{H}_{5}\right.$, furan, thiazole $\left.\mathrm{C}\right), 179.6(\mathrm{CO}), 181.3(\mathrm{C}=\mathrm{S})$. Anal. Calcd for $\mathrm{C}_{18} \mathrm{H}_{13} \mathrm{NO}_{2} \mathrm{~S}_{2}$ : C, 63.69; $\mathrm{H}, 3.86 ; \mathrm{N}, 4.13 ; \mathrm{S}$, 18.89. Found: C, $63.80 ; \mathrm{H}, 3.69 ; \mathrm{N}, 4.32 ; \mathrm{S}, 19.18$. MS: $\mathrm{m} / \mathrm{z}$ $339\left(\mathrm{M}^{+}, 48 \%\right)$.

\section{3-Phenyl-4-(thiophen-2-ylmethylene)-2-thioxo-2,3,6,7- tetrahydrobenzo $[d]$ thiazol-5(4H)-one $(8 \mathrm{~b})$ \\ Pale yellow crystals from 1,4-dioxane, yield $2.84 \mathrm{~g}$ (80\%), m.p. $222-225^{\circ} \mathrm{C}$. IR (KBr) $v_{\max }\left(\mathrm{cm}^{-1}\right) 3055(\mathrm{CH}$, aromatic), $1689(\mathrm{CO}), 1630(\mathrm{C}=\mathrm{C}), 1205(\mathrm{C}=\mathrm{S}) ;{ }^{1} \mathrm{H}$ NMR $\left(\mathrm{DMSO}-d_{6}, 300 \mathrm{MHz}\right) \delta 2.61-2.79\left(2 \mathrm{t}, 4 \mathrm{H}, 2 \mathrm{CH}_{2}\right), 6.88$ (s, $1 \mathrm{H}, \mathrm{CH}), 6.96-7.89\left(\mathrm{~m}, 8 \mathrm{H}, \mathrm{C}_{6} \mathrm{H}_{5}\right.$, thiophene $\left.\mathrm{H}\right) ;{ }^{13} \mathrm{C}$}


NMR (DMSO- $\left.d_{6}, 75 \mathrm{MHz}\right) \delta 16.5,39.7\left(2 \mathrm{CH}_{2}\right), 112.4$, $158.6(\mathrm{C}=\mathrm{CH}), 120.7,122.5,124.3,126.8,136.2,140.6$, $141.3,142.6,143.4,146.6\left(\mathrm{C}_{6} \mathrm{H}_{5}\right.$, thiophene, thiazole $\left.\mathrm{C}\right)$, $179.3(\mathrm{CO}), 181.3(\mathrm{C}=\mathrm{S})$. Anal. Calcd for $\mathrm{C}_{18} \mathrm{H}_{13} \mathrm{NOS}_{3}$ : C, 60.81; H, 3.69; N, 3.94; S, 27.06. Found: C, 60.69; H, 3.74; $\mathrm{N}, 4.12$; S, 26.86. MS: $m / z 355\left(\mathrm{M}^{+}, 59 \%\right)$.

\section{1. 5. General Procedure for the Synthesis of the 4-Hydrazono-4,5,6,7-tetrahydro- $2 \mathrm{H}$ - indazole Derivatives 10a-d}

Either hydrazine hydrate $(0.1 \mathrm{~mL}, 0.02 \mathrm{~mol})$ or phenylhydrazine $(2.16 \mathrm{~g}, 0.02 \mathrm{~mol})$ was added to a solution of either of compound $3 \mathbf{a}(1.90 \mathrm{~g}, 0.01 \mathrm{~mol})$ or $3 \mathbf{b}(2.06 \mathrm{~g}$, $0.01 \mathrm{~mol})$ in 1,4-dioxane $(40 \mathrm{~mL})$. The reaction mixture was heated under reflux for $3 \mathrm{~h}$ then poured onto ice/water mixture containing a few drops of hydrochloric acid and the formed solid product was collected by filtration.

\section{3-(Furan-2-yl)-4-hydrazono-4,5,6,7-tetrahydro-2H- indazole (10a)}

Yellow crystals from ethanol, yield $1.55 \mathrm{~g}(72 \%)$, m.p. $158-161{ }^{\circ} \mathrm{C}$. IR (KBr) $v_{\max }\left(\mathrm{cm}^{-1}\right) 3472-3328(\mathrm{NH}$, $\left.\mathrm{NH}_{2}\right), 3055(\mathrm{CH}$, aromatic), 1658 (exocyclic $\mathrm{C}=\mathrm{N}), 1630$ $(\mathrm{C}=\mathrm{C}) ;{ }^{1} \mathrm{H}$ NMR (DMSO- $\left.d_{6}, 300 \mathrm{MHz}\right) \delta 1.32-1.64(\mathrm{~m}$, $\left.2 \mathrm{H}, \mathrm{CH}_{2}\right), 2.60-2.78\left(\mathrm{~m}, 4 \mathrm{H}, 2 \mathrm{CH}_{2}\right), 4.86\left(\mathrm{~s}, 2 \mathrm{H}, \mathrm{D}_{2} \mathrm{O}\right.$ exchangeable, $\left.\mathrm{NH}_{2}\right), 7.14-7.84(\mathrm{~m}, 3 \mathrm{H}$, furan $\mathrm{H}), 8.28(\mathrm{~s}, 1 \mathrm{H}$, $\mathrm{D}_{2} \mathrm{O}$ exchangeable, $\left.\mathrm{NH}\right) ;{ }^{13} \mathrm{C}$ NMR (DMSO- $\left.d_{6}, 75 \mathrm{MHz}\right) \delta$ $16.5,36.3,39.7\left(3 \mathrm{CH}_{2}\right), 112.4,158.6(\mathrm{C}=\mathrm{CH}), 136.4,138.0$, $139.2,140.3,143.8,146.2$ (furan, pyrazole $\mathrm{C}$ ), 168.3, 172.8 $(2 \mathrm{C}=\mathrm{N})$. Anal. Calcd for $\mathrm{C}_{11} \mathrm{H}_{12} \mathrm{~N}_{4} \mathrm{O}: \mathrm{C}, 61.10 ; \mathrm{H}, 5.59$; $\mathrm{N}, 25.91$. Found: C, 60.93; H, 5.29; N, 25.77. MS: $m / z 216$ $\left(\mathrm{M}^{+}, 36 \%\right)$.

\section{3-(Furan-2-yl)-2-phenyl-4-(2-phenylhydrazono)- 4,5,6,7-tetrahydro- $2 \mathrm{H}$-indazole (10b)}

Pale yellow crystals from 1,4-dioxane, yield $2.50 \mathrm{~g}$ (68\%), m.p. $177-180^{\circ} \mathrm{C}$. IR (KBr) $v_{\max }\left(\mathrm{cm}^{-1}\right) 3463-3341$ $(\mathrm{NH}), 3055(\mathrm{CH}$, aromatic), 1652 (exocyclic $\mathrm{C}=\mathrm{N}), 1630$ $(\mathrm{C}=\mathrm{C}) ;{ }^{1} \mathrm{H}$ NMR (DMSO- $\left.d_{6}, 300 \mathrm{MHz}\right) \delta 1.31-1.67(\mathrm{~m}$, $\left.2 \mathrm{H}, \mathrm{CH}_{2}\right), 2.63-2.77\left(\mathrm{~m}, 4 \mathrm{H}, 2 \mathrm{CH}_{2}\right), 7.26-7.89(\mathrm{~m}, 13 \mathrm{H}$, $2 \mathrm{C}_{6} \mathrm{H}_{5}$, furan $\left.\mathrm{H}\right), 8.30$ (s, $1 \mathrm{H}, \mathrm{D}_{2} \mathrm{O}$ exchangeable, $\mathrm{NH}$ ); ${ }^{13} \mathrm{C}$ NMR (DMSO- $\left.d_{6}, 75 \mathrm{MHz}\right) \delta 16.2,36.1,39.4\left(3 \mathrm{CH}_{2}\right)$, $120.2,121.6,123.2,123.8,124.0,126.3,128.3,1290.1,136.4$, $140.3,140.8,141.2,143.4,146.8\left(2 \mathrm{C}_{6} \mathrm{H}_{5}\right.$, pyrazole, furan C), 176.2, $178.3(2 \mathrm{C}=\mathrm{N})$. Anal. Calcd for $\mathrm{C}_{23} \mathrm{H}_{20} \mathrm{~N}_{4} \mathrm{O}: \mathrm{C}$, 74.98; H, 5.47; N, 15.21. Found: C, 75.19; H, 5.31; N, 15.02. MS: $m / z 368\left(\mathrm{M}^{+}, 41 \%\right)$.

\section{4-Hydrazono-3-(thiophen-2-yl)-4,5,6,7-tetrahydro- $2 \mathrm{H}$ - indazole (10c)}

Pale yellow crystals from 1,4-dioxane, yield $1.76 \mathrm{~g}$ (76\%), m.p. $233-236{ }^{\circ} \mathrm{C}$. IR (KBr) $v_{\max }\left(\mathrm{cm}^{-1}\right) 3458-3342$ $\left(\mathrm{NH}, \mathrm{NH}_{2}\right), 3055(\mathrm{CH}$, aromatic), $1656($ exocyclic $\mathrm{C}=\mathrm{N})$, $1630(\mathrm{C}=\mathrm{C}) ;{ }^{1} \mathrm{H}$ NMR (DMSO- $\left.d_{6}, 300 \mathrm{MHz}\right) \delta 1.33-1.67$ $\left(\mathrm{m}, 2 \mathrm{H}, \mathrm{CH}_{2}\right), 2.62-2.75\left(\mathrm{~m}, 4 \mathrm{H}, 2 \mathrm{CH}_{2}\right), 4.80\left(\mathrm{~s}, 2 \mathrm{H}, \mathrm{D}_{2} \mathrm{O}\right.$ exchangeable, $\left.\mathrm{NH}_{2}\right), 6.96-7.88(\mathrm{~m}, 3 \mathrm{H}$, thiophene $\mathrm{H}), 8.28$ $\left(\mathrm{s}, 1 \mathrm{H}, \mathrm{D}_{2} \mathrm{O}\right.$ exchangeable, $\left.\mathrm{NH}\right) ;{ }^{13} \mathrm{C}$ NMR (DMSO- $d_{6}$, $75 \mathrm{MHz}) \delta 16.5,36.3,39.7\left(3 \mathrm{CH}_{2}\right), 112.4,158.6(\mathrm{C}=\mathrm{CH})$, 136.6, 138.4, 139.2, 140.1, 143.5, 146.4 (thiphene, pyrazole C), 176.4, $178.6(2 \mathrm{C}=\mathrm{N})$. Anal. Calcd for $\mathrm{C}_{11} \mathrm{H}_{12} \mathrm{~N}_{4} \mathrm{~S}$ : C, 56.87; H, 5.21; N, 24.12; S, 13.80. Found: C, 56.93; H, 5.30; $\mathrm{N}, 24.31 ; \mathrm{S}, 13.98$. MS: $m / z 232\left(\mathrm{M}^{+}, 28 \%\right)$.

\section{2-Phenyl-4-(2-phenylhydrazono)-3-(thiophen-2-yl)- 4,5,6,7-tetrahydro- $2 \mathrm{H}$-indazole $(10 \mathrm{~d})$}

Orange crystals from ethanol, yield $2.61 \mathrm{~g}(68 \%)$, m.p. $180-184^{\circ} \mathrm{C}$. IR $(\mathrm{KBr}) v_{\max }\left(\mathrm{cm}^{-1}\right) 3484-3326(\mathrm{NH})$, $3055(\mathrm{CH}$, aromatic), 1655 (exocyclic $\mathrm{C}=\mathrm{N}), 1630(\mathrm{C}=\mathrm{C})$; ${ }^{1} \mathrm{H}$ NMR (DMSO- $\left.d_{6}, 300 \mathrm{MHz}\right) \delta 1.32-1.69\left(\mathrm{~m}, 2 \mathrm{H}, \mathrm{CH}_{2}\right)$, 2.60-2.78 (m, $\left.4 \mathrm{H}, 2 \mathrm{CH}_{2}\right), 7.23-7.85\left(\mathrm{~m}, 13 \mathrm{H}, 2 \mathrm{C}_{6} \mathrm{H}_{5}\right.$, thiophene $\mathrm{H}$ ), 8.29 (s, $1 \mathrm{H}, \mathrm{D}_{2} \mathrm{O}$ exchangeable, $\left.\mathrm{NH}\right) ;{ }^{13} \mathrm{C}$ NMR $\left(\mathrm{DMSO}-d_{6}, 75 \mathrm{MHz}\right) \delta 16.4,36.6,39.4\left(3 \mathrm{CH}_{2}\right), 120.8$, $122.2,123.6,123.9,125.8,126.6,128.1,129.7,135.8,140.6$, $142.6,142.9,143.1,146.5\left(2 \mathrm{C}_{6} \mathrm{H}_{5}\right.$, pyrazole, thiophene $\left.\mathrm{C}\right)$, 176.4, $178.6(2 \mathrm{C}=\mathrm{N})$. Anal. Calcd for $\mathrm{C}_{23} \mathrm{H}_{20} \mathrm{~N}_{4} \mathrm{~S}: \mathrm{C}$, 71.85; H, 5.24; N, 14.57; S, 8.34. Found: C, 72.13; H, 5.42; N, 14.38; S, 8.62. MS: $m / z 384\left(\mathrm{M}^{+}, 26 \%\right)$.

\section{1. 6. General Procedure for the Synthesis of the 6,7-Dihydrobenzo[ $[c]$ isoxazol-4(5H)-one Oxime Derivatives 12a,b}

Hydroxylamine hydrochloride (1.40 g, $0.02 \mathrm{~mol})$ was added to a solution of either compound $3 \mathrm{a}$ ( $1.90 \mathrm{~g}$, $0.01 \mathrm{~mol})$ or $3 \mathbf{b}(2.06 \mathrm{~g}, 0.01 \mathrm{~mol})$ in 1,4-dioxane $(40 \mathrm{~mL})$ containing sodium acetate $(2.0 \mathrm{~g})$. The reaction mixture was heated under reflux for $3 \mathrm{~h}$ then poured onto ice/water mixture and the formed solid product, in each case, was collected by filtration.

\section{3-(Furan-2-yl)-6,7-dihydrobenzo[c]isoxazol-4(5H)-one Oxime (12a)}

Yellow crystals from 1,4-dioxane, yield $1.52 \mathrm{~g}(70 \%)$, m.p. $177-180^{\circ} \mathrm{C}$. IR $(\mathrm{KBr}) v_{\max }\left(\mathrm{cm}^{-1}\right) 3524-3376(\mathrm{OH})$, $3055(\mathrm{CH}$, aromatic), 1655 (exocyclic $\mathrm{C}=\mathrm{N}), 1630(\mathrm{C}=\mathrm{C})$; ${ }^{1} \mathrm{H}$ NMR (DMSO- $\left.d_{6}, 300 \mathrm{MHz}\right) \delta 1.31-1.68\left(\mathrm{~m}, 2 \mathrm{H}, \mathrm{CH}_{2}\right)$, 2.63-2.77 (m, $\left.4 \mathrm{H}, 2 \mathrm{CH}_{2}\right), 6.93-7.81(\mathrm{~m}, 3 \mathrm{H}$, furan $\mathrm{H})$, 10.29 (s, $1 \mathrm{H}, \mathrm{D}_{2} \mathrm{O}$ exchangeable, $\left.\mathrm{OH}\right) ;{ }^{13} \mathrm{C}$ NMR (DMSO- $\left.d_{6}, 75 \mathrm{MHz}\right) \delta 16.2,36.3,39.2\left(3 \mathrm{CH}_{2}\right), 132.3,135.1$, 140.6, 141.5, 142.8, 143.6 (isoxazole, furan C), 176.1, 178.4 $(2 \mathrm{C}=\mathrm{N})$. Anal. Calcd for $\mathrm{C}_{11} \mathrm{H}_{10} \mathrm{~N}_{2} \mathrm{O}_{3}: \mathrm{C}, 60.55 ; \mathrm{H}, 4.62$; N, 12.84. Found: C, 60.39; H, 4.80; N, 12.93. MS: $m / z 218$ $\left(\mathrm{M}^{+}, 32 \%\right)$.

3-(Thiophen-2-yl)-6,7-dihydrobenzo[c]isoxazol-4(5H)one Oxime (12b)

Pale yellow crystals from 1,4-dioxane, yield $1.52 \mathrm{~g}$ (65\%), m.p. $222-225^{\circ} \mathrm{C}$. IR (KBr) $v_{\max }\left(\mathrm{cm}^{-1}\right) 3551-3349$ $(\mathrm{OH}), 3055(\mathrm{CH}$, aromatic), 1653 (exocyclic $\mathrm{C}=\mathrm{N}), 1630$ $(\mathrm{C}=\mathrm{C}) ;{ }^{1} \mathrm{H}$ NMR (DMSO- $\left.d_{6}, 300 \mathrm{MHz}\right) \delta 1.32-1.65(\mathrm{~m}$, $\left.2 \mathrm{H}, \mathrm{CH}_{2}\right), 2.61-2.74\left(\mathrm{~m}, 4 \mathrm{H}, 2 \mathrm{CH}_{2}\right), 7.22-7.80(\mathrm{~m}, 3 \mathrm{H}$, 
thiophene $\mathrm{H}), 10.29$ (s, $1 \mathrm{H}, \mathrm{D}_{2} \mathrm{O}$ exchangeable, $\left.\mathrm{OH}\right) ;{ }^{13} \mathrm{C}$ NMR (DMSO- $\left.d_{6}, 75 \mathrm{MHz}\right) \delta 16.4,36.6,39.8\left(3 \mathrm{CH}_{2}\right), 132.1$, 134.3, 138.8, 140.9, 141.6, 144.2 (isoxazole, thiophene C), 176.0, $178.6(2 \mathrm{C}=\mathrm{N})$. Anal. Calcd for $\mathrm{C}_{11} \mathrm{H}_{10} \mathrm{~N}_{2} \mathrm{O}_{2} \mathrm{~S}: \mathrm{C}$, 56.39; H, 4.30; N, 11.96; S, 13.69. Found: C, 56.42; H, 4.49; $\mathrm{N}, 12.05 ; \mathrm{S}, 13.83 \mathrm{MS}: \mathrm{m} / z 234\left(\mathrm{M}^{+}, 42 \%\right)$.

\section{1. 7. 2-(Ethoxymethylene)cyclohexane-1,3- dione (14)}

Ethyl orthoformate $(1.68 \mathrm{~g}, 0.01 \mathrm{~mol})$ was added to a solution of cyclohexan-1,3-dione (1.12 g, $0.01 \mathrm{~mol})$ in acetic acid $(40 \mathrm{~mL})$. The reaction mixture was heated under reflux for $2 \mathrm{~h}$ then evaporated in vacuum and the remaining product was triturated with ethanol and the formed solid product was collected by filtration.

Yellow crystals from ethanol, yield $1.27 \mathrm{~g}(76 \%)$, m.p. $282-258^{\circ} \mathrm{C}$. IR $(\mathrm{KBr}) v_{\max }\left(\mathrm{cm}^{-1}\right) 2980\left(\mathrm{CH}_{2}\right), 1689$, 1686 (CO), $1632(\mathrm{C}=\mathrm{C}) ;{ }^{1} \mathrm{H}$ NMR (DMSO- $d_{6}, 300 \mathrm{MHz}$ ) $\delta 1.28\left(\mathrm{t}, 3 \mathrm{H}, J=6.83 \mathrm{~Hz}, \mathrm{CH}_{3}\right), 1.49-1.67\left(\mathrm{~m}, 2 \mathrm{H}, \mathrm{CH}_{2}\right)$, $2.65-2.73\left(\mathrm{~m}, 4 \mathrm{H}, 2 \mathrm{CH}_{2}\right), 3.89\left(\mathrm{q}, 2 \mathrm{H}, J=6.83 \mathrm{~Hz}, \mathrm{CH}_{2}\right)$, $6.79\left(\mathrm{~s}, 1 \mathrm{H}, \mathrm{CH}\right.$ ); ${ }^{13} \mathrm{C}$ NMR (DMSO- $\left.d_{6}, 75 \mathrm{MHz}\right) \delta 16.3$ $\left(\mathrm{OCH}_{2} \mathrm{CH}_{3}\right), 16.2,36.8,39.0\left(3 \mathrm{CH}_{2}\right), 62.8\left(\mathrm{OCH}_{2} \mathrm{CH}_{3}\right)$, 112.3, 158.2 $(\mathrm{C}=\mathrm{CH}), 177.3,179.2(2 \mathrm{CO})$. Analysis Calcd for $\mathrm{C}_{9} \mathrm{H}_{12} \mathrm{O}_{3}$ (168.19): C, 64.27; H, 7.19\%. Found: C, 64.08; $\mathrm{H}, 7.33 \%$. MS: $m / z 168\left(\mathrm{M}^{+}, 22 \%\right)$.

\section{1. 8. General Procedure for the Synthesis of the 2-(Aminomethylene) cyclohexane-1,3- dione Derivatives 16a-c}

Equimolar amounts of aniline $(0.93 \mathrm{~g}, 0.01 \mathrm{~mol})$, 4-methylaniline (1.08 g, $0.01 \mathrm{~mol})$ or 4-methoxyaniline $(1.34 \mathrm{~g}, 0.01 \mathrm{~mol})$ and compound $14(1.68 \mathrm{~g}, 0.01 \mathrm{~mol})$ in 1,4-dioxane $(50 \mathrm{~mL})$ were refluxed for $4 \mathrm{~h}$. The reaction mixture was evaporated under vacuum and the remaining product was triturated with ethanol and the solid product formed, in each case, was collected by filtration.

\section{2-((Phenylamino)methylene)cyclohexane-1,3-dione (16a)}

Yellow crystals from ethanol, yield $1.24 \mathrm{~g}(58 \%)$, m.p. $165-167^{\circ} \mathrm{C}$. IR (KBr) $v_{\max }\left(\mathrm{cm}^{-1}\right) 3480-3363(\mathrm{NH})$, 3055 ( $\mathrm{CH}$ aromatic), $2980\left(\mathrm{CH}_{2}\right), 1688,1686(2 \mathrm{CO}), 1632$ $(\mathrm{C}=\mathrm{C}) ;{ }^{1} \mathrm{H}$ NMR (DMSO- $\left.d_{6}, 300 \mathrm{MHz}\right) \delta 1.42-1.69(\mathrm{~m}$, $\left.2 \mathrm{H}, \mathrm{CH}_{2}\right), 2.63-2.75\left(\mathrm{~m}, 4 \mathrm{H}, 2 \mathrm{CH}_{2}\right), 6.05(\mathrm{~s}, 1 \mathrm{H}, \mathrm{CH})$, 7.28-7.39 (s, $\left.5 \mathrm{H}, \mathrm{C}_{6} \mathrm{H}_{5}\right), 8.28\left(\mathrm{~s}, 1 \mathrm{H}, \mathrm{D}_{2} \mathrm{O}\right.$ exchangeable, $\mathrm{NH}) ;{ }^{13} \mathrm{C}$ NMR (DMSO- $\left.d_{6}, 75 \mathrm{MHz}\right) \delta 16.5,36.8,39.4$ $\left(3 \mathrm{CH}_{2}\right), 94.3(\mathrm{CH}), 120.3,123.1,124.8,126.4\left(\mathrm{C}_{6} \mathrm{H}_{5}\right)$, 177.1, 179.8 (2CO). Anal. Calcd for $\mathrm{C}_{13} \mathrm{H}_{13} \mathrm{NO}_{2}$ (215.25): C, 72.54; H, 6.09; N, 6.51\%. Found: C, 72.31; H, 6.29; N, 6.38\%.\%. MS: $m / z 215\left(\mathrm{M}^{+}, 35 \%\right)$.

\footnotetext{
2-((Phenylamino)methylene)cyclohexane-1,3-dione (16b)

Yellow crystals from ethanol, yield $1.67 \mathrm{~g}(73 \%)$, m.p. 214-217 ${ }^{\circ} \mathrm{C}$. IR (KBr) $v_{\max }\left(\mathrm{cm}^{-1}\right) 3459-3341(\mathrm{NH}), 3054$ (CH aromatic), 2982, $2886\left(\mathrm{CH}_{3}, \mathrm{CH}_{2}\right), 1688,1686$ (2CO),
}

$1631(\mathrm{C}=\mathrm{C}) ;{ }^{1} \mathrm{H}$ NMR (DMSO- $\left.d_{6}, 300 \mathrm{MHz}\right) \delta 1.43-1.67$ $\left(\mathrm{m}, 2 \mathrm{H}, \mathrm{CH}_{2}\right), 2.61-2.76\left(\mathrm{~m}, 4 \mathrm{H}, 2 \mathrm{CH}_{2}\right), 2.88\left(\mathrm{~s}, 3 \mathrm{H}, \mathrm{CH}_{3}\right)$, $6.08(\mathrm{~s}, 1 \mathrm{H}, \mathrm{CH}), 7.24-7.45\left(\mathrm{~s}, 4 \mathrm{H}, \mathrm{C}_{6} \mathrm{H}_{4}\right), 8.26\left(\mathrm{~s}, 1 \mathrm{H}, \mathrm{D}_{2} \mathrm{O}\right.$ exchangeable, $\mathrm{NH}$ ); ${ }^{13} \mathrm{C}$ NMR (DMSO- $\left.d_{6}, 75 \mathrm{MHz}\right) \delta 16.3$, 36.6, $39.5\left(3 \mathrm{CH}_{2}\right), 23.8\left(\mathrm{CH}_{3}\right), 90.6(\mathrm{CH}), 120.8,122.9$, 123.4, 127.9 $\left(\mathrm{C}_{6} \mathrm{H}_{4}\right), 177.3,179.5(2 \mathrm{CO})$. Analysis Calcd for $\mathrm{C}_{14} \mathrm{H}_{15} \mathrm{NO}_{2}$ (229.27): C, 73.34; H, 6.59; N, 6.11\%. Found: C, 73.29; H, 6.41; N, 6.26\%. MS: $m / z 229\left(\mathrm{M}^{+}, 40 \%\right)$.

\section{2-((4-Methoxyphenyl)amino)methylene)cyclohexane-} 1,3-dione (16c)

Yellow crystals from 1,4-dioxane, yield $1.47 \mathrm{~g}(60 \%)$, m.p. $193-196^{\circ} \mathrm{C}$. IR $(\mathrm{KBr}) v_{\max }\left(\mathrm{cm}^{-1}\right) 3463-3329(\mathrm{NH})$, 3055 (CH aromatic), 2982, $2886\left(\mathrm{CH}_{3}, \mathrm{CH}_{2}\right), 1689,1686$ (2CO), $1630(\mathrm{C}=\mathrm{C}) ;{ }^{1} \mathrm{H}$ NMR (DMSO- $\left.d_{6}, 300 \mathrm{MHz}\right) \delta$ 1.41-1.69 (m, $\left.2 \mathrm{H}, \mathrm{CH}_{2}\right), 2.61-2.78\left(\mathrm{~m}, 4 \mathrm{H}, 2 \mathrm{CH}_{2}\right), 3.68(\mathrm{~s}$, $\left.3 \mathrm{H}, \mathrm{OCH}_{3}\right), 6.06(\mathrm{~s}, 1 \mathrm{H}, \mathrm{CH}), 7.27-7.48\left(\mathrm{~s}, 4 \mathrm{H}, \mathrm{C}_{6} \mathrm{H}_{4}\right), 8.28$ (s, $1 \mathrm{H}, \mathrm{D}_{2} \mathrm{O}$ exchangeable, $\mathrm{NH}$ ); ${ }^{13} \mathrm{C}$ NMR (DMSO- $d_{6}, 75$ $\mathrm{MHz}) \delta 16.3,36.6,39.5\left(3 \mathrm{CH}_{2}\right), 50.3\left(\mathrm{OCH}_{3}\right), 90.2(\mathrm{CH})$, 120.3, 122.4, 125.6, $128.7\left(\mathrm{C}_{6} \mathrm{H}_{4}\right), 177.1,179.3$ (2CO). Analysis Calcd for $\mathrm{C}_{14} \mathrm{H}_{15} \mathrm{NO}_{3}$ (245.27): C, 68.56; H, 6.16; N, 5.71\%. Found: C, 68.80; H, 6.24; N, 5.93\%. MS: $m / z 245$ $\left(\mathrm{M}^{+}, 38 \%\right)$.

\section{1. 9. General Procedure for the Synthesis of the 6,7-Dihydrobenzo $[b]$ thiophen-5(4H)-one Derivatives 17a-f}

Either malononitrile $(0.66 \mathrm{~g}, 0.01 \mathrm{~mol})$ or ethyl cyanoacetate $(1.07 \mathrm{~g}, 0.01 \mathrm{~mol})$ was added to a solution of either compound 16a (2.15 g, $0.01 \mathrm{~mol}), \mathbf{1 6 b}(2.29 \mathrm{~g}, 0.01$ $\mathrm{mol})$ or $16 \mathrm{c}(2.45 \mathrm{~g}, 0.01 \mathrm{~mol})$ in ethanol $(40 \mathrm{~mL})$ containing triethylamine $(0.50 \mathrm{~mL})$. The reaction mixture was heated under reflux for $3 \mathrm{~h}$ then poured onto ice/water mixture containing a few drops of hydrochloric acid and the formed solid product was collected by filtration.

\section{2-Amino-5-oxo-4-((phenylamino)methylene)-4,5,6,7- tetrahydrobenzo $[b]$ thiophene-3-carbonitrile (17a)}

Pale yellow crystals from ethanol, yield $2.06 \mathrm{~g}(70 \%)$, m.p. $127-129^{\circ} \mathrm{C}$. IR $(\mathrm{KBr}) v_{\max }\left(\mathrm{cm}^{-1}\right) 3472-3353\left(\mathrm{NH}_{2}\right.$, $\mathrm{NH}), 3055(\mathrm{CH}$, aromatic), $2220(\mathrm{CN}), 1686(\mathrm{CO}), 1630$ $(\mathrm{C}=\mathrm{C}) ;{ }^{1} \mathrm{H}$ NMR (DMSO- $\left.d_{6}, 300 \mathrm{MHz}\right) \delta 2.60-2.79(2 \mathrm{t}$, $\left.4 \mathrm{H}, 2 \mathrm{CH}_{2}\right), 4.78\left(\mathrm{~s}, 2 \mathrm{H}, \mathrm{D}_{2} \mathrm{O}\right.$ exchangeable, $\left.\mathrm{NH}_{2}\right), 6.89(\mathrm{~s}$, $1 \mathrm{H}, \mathrm{CH}), 7.26-7.42\left(\mathrm{~m}, 5 \mathrm{H}, \mathrm{C}_{6} \mathrm{H}_{5}\right), 8.39\left(\mathrm{~s}, 1 \mathrm{H}, \mathrm{D}_{2} \mathrm{O}\right.$ exchangeable, $\mathrm{NH}$ ); ${ }^{13} \mathrm{C}$ NMR (DMSO- $\left.d_{6}, 75 \mathrm{MHz}\right) \delta 36.0$, $39.8\left(2 \mathrm{CH}_{2}\right), 112.2,158.4(\mathrm{C}=\mathrm{CH}), 116.8(\mathrm{CN}), 121.6$, $122.4,124.8,127.2,132.7,134.2,138.0,142.6\left(\mathrm{C}_{6} \mathrm{H}_{5}\right.$, thiophene C), $179.6(\mathrm{CO})$. Anal. Calcd for $\mathrm{C}_{16} \mathrm{H}_{13} \mathrm{~N}_{3} \mathrm{OS}$ : C, 65.06; H, 4.44; N, 14.23; S, 10.86. Found: C, 65.18; H, 4.60; $\mathrm{N}, 14.19 ; \mathrm{S}, 11.17$. MS: $\mathrm{m} / z 295\left(\mathrm{M}^{+}, 38 \%\right)$.

Ethyl 2-Amino-5-oxo-4-((phenylamino)methylene)4,5,6,7-tetrahydrobenzo $[b]$ thiophene-3-carboxylate (17b) Yellow crystals from ethanol, yield $2.18 \mathrm{~g}(64 \%), \mathrm{m} . \mathrm{p}$. 96-98 ${ }^{\circ} \mathrm{C}$. IR ( $\left.\mathrm{KBr}\right) v_{\max }\left(\mathrm{cm}^{-1}\right) 3486-3351\left(\mathrm{NH}_{2}\right), 3058$ 
$\left(\mathrm{CH}\right.$, aromatic), 2930, $2972\left(\mathrm{CH}_{2}, \mathrm{CH}_{3}\right), 1689,1686(2$ CO), $1630(\mathrm{C}=\mathrm{C}) ;{ }^{1} \mathrm{H}$ NMR (DMSO- $\left.d_{6}, 300 \mathrm{MHz}\right) \delta 1.13$ (t, $\left.3 \mathrm{H}, J=7.08 \mathrm{~Hz}, \mathrm{CH}_{3}\right), 2.68-2.76\left(2 \mathrm{t}, 4 \mathrm{H}, 2 \mathrm{CH}_{2}\right), 4.22$ $\left(\mathrm{q}, 2 \mathrm{H}, J=7.08 \mathrm{~Hz}, \mathrm{CH}_{2}\right), 4.77\left(\mathrm{~s}, 2 \mathrm{H}, \mathrm{D}_{2} \mathrm{O}\right.$ exchangeable, $\left.\mathrm{NH}_{2}\right), 6.86(\mathrm{~s}, 1 \mathrm{H}, \mathrm{CH}), 7.22-7.40\left(\mathrm{~m}, 5 \mathrm{H}, \mathrm{C}_{6} \mathrm{H}_{5}\right), 8.33$ (s, $1 \mathrm{H}, \mathrm{D}_{2} \mathrm{O}$ exchangeable, $\mathrm{NH}$ ); ${ }^{13} \mathrm{C}$ NMR (DMSO- $d_{6}$, $75 \mathrm{MHz}) \delta 16.3\left(\mathrm{OCH}_{2} \mathrm{CH}_{3}\right), 36.8,39.6\left(2 \mathrm{CH}_{2}\right), 52.5$ $\left(\mathrm{OCH}_{2} \mathrm{CH}_{3}\right), 112.4,158.8(\mathrm{C}=\mathrm{CH}), 121.3,123.6,125.2$, $126.8,132.9,133.3,138.0,142.7\left(\mathrm{C}_{6} \mathrm{H}_{5}\right.$, thiohene $\left.\mathrm{C}\right), 164.2$, 179.8 (2CO). Anal. Calcd for $\mathrm{C}_{18} \mathrm{H}_{18} \mathrm{~N}_{2} \mathrm{O}_{3} \mathrm{~S}: \mathrm{C}, 63.14 ; \mathrm{H}$, 5.30; N, 8.18; S, 9.36. Found: C, 62.91; H, 5.49; N, 8.25; S, 9.60. MS: $m / z 342\left(\mathrm{M}^{+}, 27 \%\right)$.

\section{2-Amino-5-oxo-4-(( $p$-tolylamino) methylene)-4,5,6,7- tetrahydrobenzo- $[c]$ thiophene-3-carbonitrile $(17 \mathrm{c})$} Orange crystals from 1,4-dioxane, yield $2.31 \mathrm{~g}(75 \%)$, m.p. $177-179^{\circ} \mathrm{C}$. IR (KBr) $v_{\max }\left(\mathrm{cm}^{-1}\right) 3493-3323\left(\mathrm{NH}_{2}\right.$, $\mathrm{NH}), 3055(\mathrm{CH}$, aromatic), $2220(\mathrm{CN}), 1688(\mathrm{CO}), 1630$ $(\mathrm{C}=\mathrm{C}) ;{ }^{1} \mathrm{H}$ NMR (DMSO- $\left.d_{6}, 300 \mathrm{MHz}\right) \delta 2.62-2.77(2 \mathrm{t}$, $\left.4 \mathrm{H}, 2 \mathrm{CH}_{2}\right), 2.79\left(\mathrm{~s}, 3 \mathrm{H}, \mathrm{CH}_{3}\right), 4.77\left(\mathrm{~s}, 2 \mathrm{H}, \mathrm{D}_{2} \mathrm{O}\right.$ exchangeable, $\left.\mathrm{NH}_{2}\right), 6.82(\mathrm{~s}, 1 \mathrm{H}, \mathrm{CH}), 7.28-7.46\left(\mathrm{~m}, 4 \mathrm{H}, \mathrm{C}_{6} \mathrm{H}_{4}\right), 8.37$ (s, $1 \mathrm{H}, \mathrm{D}_{2} \mathrm{O}$ exchangeable, $\mathrm{NH}$ ); ${ }^{13} \mathrm{C}$ NMR (DMSO- $d_{6}$, $75 \mathrm{MHz}) \delta 36.0,39.6\left(2 \mathrm{CH}_{2}\right), 24.3\left(\mathrm{CH}_{3}\right), 112.0,158.6$ $(\mathrm{C}=\mathrm{CH}), 116.9(\mathrm{CN}), 120.3,123.7,124.8,128.9,132.9$, 134.7, 138.3, $142.2\left(\mathrm{C}_{6} \mathrm{H}_{4}\right.$, thiophene $\left.\mathrm{C}\right), 179.3$ (CO). Anal. Calcd for $\mathrm{C}_{17} \mathrm{H}_{15} \mathrm{~N}_{3} \mathrm{OS}$ : C, 66.00; $\mathrm{H}, 4.89 ; \mathrm{N}, 13.58$; S, 10.36. Found: C, 65.85; H, 4.91; N, 13.70; S, 10.42. MS: $\mathrm{m} / z 309\left(\mathrm{M}^{+}, 24 \%\right)$.

Ethyl 2-Amino-5-oxo-4-((p-tolylamino)methylene)4,5,6,7-tetrahydrobenzo $[b]$ thiophene-3-carboxylate (17d)

Brown crystals from 1,4-dioxane, yield $2.45 \mathrm{~g}(69 \%)$, m.p. 203-206 ${ }^{\circ} \mathrm{C}$. IR $(\mathrm{KBr}) v_{\max }\left(\mathrm{cm}^{-1}\right) 3459-3337\left(\mathrm{NH}_{2}\right)$, $3055\left(\mathrm{CH}\right.$, aromatic), 2930, $2970\left(\mathrm{CH}_{2}, \mathrm{CH}_{3}\right), 1689,1687$ (2 CO), $1630(\mathrm{C}=\mathrm{C}) ;{ }^{1} \mathrm{H}$ NMR (DMSO- $\left.d_{6}, 300 \mathrm{MHz}\right) \delta$ $1.12\left(\mathrm{t}, 3 \mathrm{H}, J=6.59 \mathrm{~Hz}, \mathrm{CH}_{3}\right), 2.65-2.78\left(2 \mathrm{t}, 4 \mathrm{H}, 2 \mathrm{CH}_{2}\right)$, $2.80\left(\mathrm{~s}, 3 \mathrm{H}, \mathrm{CH}_{3}\right), 4.24\left(\mathrm{q}, 2 \mathrm{H}, J=6.59 \mathrm{~Hz}, \mathrm{CH}_{2}\right), 4.79(\mathrm{~s}$, $2 \mathrm{H}, \mathrm{D}_{2} \mathrm{O}$ exchangeable, $\left.\mathrm{NH}_{2}\right), 6.83(\mathrm{~s}, 1 \mathrm{H}, \mathrm{CH}), 7.21-7.45$ $\left(\mathrm{m}, 4 \mathrm{H}, \mathrm{C}_{6} \mathrm{H}_{4}\right), 8.36\left(\mathrm{~s}, 1 \mathrm{H}, \mathrm{D}_{2} \mathrm{O}\right.$ exchangeable, $\left.\mathrm{NH}\right) ;{ }^{13} \mathrm{C}$ NMR (DMSO- $\left.d_{6}, 75 \mathrm{MHz}\right) \delta 16.1\left(\mathrm{OCH}_{2} \mathrm{CH}_{3}\right), 36.3$, $39.7\left(3 \mathrm{CH}_{2}\right), 23.9\left(\mathrm{CH}_{3}\right), 52.1\left(\mathrm{OCH}_{2} \mathrm{CH}_{3}\right), 112.2,158.7$ $(\mathrm{C}=\mathrm{CH}), 120.1,123.8,124.9,128.3,134.8,137.2,140.2$, $142.6\left(\mathrm{C}_{6} \mathrm{H}_{5}\right.$, thiophene C), 164.3, $179.5(2 \mathrm{CO})$. Anal. Calcd for $\mathrm{C}_{19} \mathrm{H}_{20} \mathrm{~N}_{2} \mathrm{O}_{3} \mathrm{~S}$ : C, 64.02; H, 5.66; N, 7.86; S, 9.00. Found: C, 63.91; H, 5.53; N, 8.01; S, 9.26. MS: $m / z 356\left(\mathrm{M}^{+}\right.$, $32 \%)$.

\section{2-Amino-4-((4-methoxyphenyl)amino)methylene)- 5-oxo-4,5,6,7-tetrahydrobenzo $[b]$ thiophene-3- carbonitrile (17e)}

Yellow crystals from ethanol, yield $2.30 \mathrm{~g}(71 \%)$, m.p. 166-169 ${ }^{\circ} \mathrm{C}$. IR $(\mathrm{KBr}) v_{\max }\left(\mathrm{cm}^{-1}\right) 3474-3329\left(\mathrm{NH}_{2}, \mathrm{NH}\right)$, $3055(\mathrm{CH}$, aromatic), $2220(\mathrm{CN}), 1688(\mathrm{CO}), 1630(\mathrm{C}=\mathrm{C})$; ${ }^{1} \mathrm{H}$ NMR (DMSO- $\left.d_{6}, 300 \mathrm{MHz}\right) \delta 2.61-2.77\left(2 \mathrm{t}, 4 \mathrm{H}, 2 \mathrm{CH}_{2}\right)$, $3.68\left(\mathrm{~s}, 3 \mathrm{H}, \mathrm{OCH}_{3}\right), 4.75\left(\mathrm{~s}, 2 \mathrm{H}, \mathrm{D}_{2} \mathrm{O}\right.$ exchangeable, $\left.\mathrm{NH}_{2}\right)$, $6.87(\mathrm{~s}, 1 \mathrm{H}, \mathrm{CH}), 7.24-7.46\left(\mathrm{~m}, 4 \mathrm{H}, \mathrm{C}_{6} \mathrm{H}_{4}\right), 8.35(\mathrm{~s}, 1 \mathrm{H}$, $\mathrm{D}_{2} \mathrm{O}$ exchangeable, $\mathrm{NH}$ ); ${ }^{13} \mathrm{C}$ NMR (DMSO- $\left.d_{6}, 75 \mathrm{MHz}\right) \delta$ $16.1,36.2,39.4\left(3 \mathrm{CH}_{2}\right), 50.2\left(\mathrm{OCH}_{3}\right), 112.5,158.4(\mathrm{C}=\mathrm{CH})$, $116.7(\mathrm{CN}), 120.1,122.9,125.3,127.6,132.2,134.7,137.6$, $143.3\left(\mathrm{C}_{6} \mathrm{H}_{4}\right.$, thiophene C), 179.6 (CO). Anal. Calcd for $\mathrm{C}_{17} \mathrm{H}_{15} \mathrm{~N}_{3} \mathrm{O}_{2} \mathrm{~S}: \mathrm{C}, 62.75 ; \mathrm{H}, 4.65 ; \mathrm{N}, 12.91 ; \mathrm{S}, 9.85$. Found: $\mathrm{C}$, 62.53; H, 4.79; N, 12.83; S, 10.01. MS: $\mathrm{m} / z 325\left(\mathrm{M}^{+}, 32 \%\right)$.

\section{Ethyl 2-Amino-4-(((4-methoxyphenyl)amino) methylene)-5-oxo-4,5,6,7-tetrahydrobenzo $[b]$ - thiophene-3-carboxylate (17f)}

Yellow crystals from ethanol, yield $2.40 \mathrm{~g}(65 \%)$, m.p. 203-206 ${ }^{\circ} \mathrm{C}$. IR (KBr) $v_{\max }\left(\mathrm{cm}^{-1}\right) 3459-3337\left(\mathrm{NH}_{2}\right)$, $3055\left(\mathrm{CH}\right.$, aromatic), 2930, $2970\left(\mathrm{CH}_{2}, \mathrm{CH}_{3}\right), 1689,1687$ (2 CO), $1630(\mathrm{C}=\mathrm{C}) ;{ }^{1} \mathrm{H}$ NMR (DMSO- $\left.d_{6}, 300 \mathrm{MHz}\right) \delta$ $1.13\left(\mathrm{t}, 3 \mathrm{H}, J=7.59 \mathrm{~Hz}, \mathrm{CH}_{3}\right), 2.62-2.75\left(2 \mathrm{t}, 4 \mathrm{H}, 2 \mathrm{CH}_{2}\right)$, $3.61\left(\mathrm{~s}, 3 \mathrm{H}, \mathrm{OCH}_{3}\right), 4.22\left(\mathrm{q}, 2 \mathrm{H}, J=7.59 \mathrm{~Hz}, \mathrm{CH}_{2}\right), 4.79$ (s, 2H, $\mathrm{D}_{2} \mathrm{O}$ exchangeable, $\left.\mathrm{NH}_{2}\right), 6.83(\mathrm{~s}, 1 \mathrm{H}, \mathrm{CH}), 7.24-$ $7.48\left(\mathrm{~m}, 4 \mathrm{H}, \mathrm{C}_{6} \mathrm{H}_{4}\right), 8.34\left(\mathrm{~s}, 1 \mathrm{H}, \mathrm{D}_{2} \mathrm{O}\right.$ exchangeable, $\left.\mathrm{NH}\right)$; ${ }^{13} \mathrm{C}$ NMR (DMSO- $\left.d_{6}, 75 \mathrm{MHz}\right) \delta 16.1\left(\mathrm{OCH}_{2} \mathrm{CH}_{3}\right), 36.3$, $39.7\left(3 \mathrm{CH}_{2}\right), 50.2\left(\mathrm{OCH}_{3}\right), 52.2\left(\mathrm{OCH}_{2} \mathrm{CH}_{3}\right), 112.2,158.7$ $(\mathrm{C}=\mathrm{CH}), 120.2,122.2,124.3,128.1,134.6,136.7,138.1$, $140.8\left(\mathrm{C}_{6} \mathrm{H}_{5}\right.$, thiophene C), 164.1, 179.7 (2CO). Anal. Calcd for $\mathrm{C}_{19} \mathrm{H}_{20} \mathrm{~N}_{2} \mathrm{O}_{4} \mathrm{~S}: \mathrm{C}, 61.27 ; \mathrm{H}, 5.41 ; \mathrm{N}, 7.52 ; \mathrm{S}, 8.61$. Found: C, 60.91; H, 5.23; N, 7.39; S, 8.59. MS: m/z $372\left(\mathrm{M}^{+}, 36 \%\right)$.

\section{1. 10. General Procedure for the Synthesis of the 6,7-Dihydrobenzo[b]thiophen-3- carboxamide Derivatives 19a-c}

Either of compounds $18 \mathrm{a}(1.12 \mathrm{~g}, 0.01 \mathrm{~mol}), \mathbf{1 8 b}$ $(1.74 \mathrm{~g}, 0.01 \mathrm{~mol})$ or $18 \mathrm{c}(1.94 \mathrm{~g}, 0.01 \mathrm{~mol})$ was added to a solution of cyclohexane-1,3-dione (1) $(1.12 \mathrm{~g}, 0.01 \mathrm{~mol})$ in 1,4-dioxane $(40 \mathrm{~mL})$ containing triethylamine $(0.50 \mathrm{~mL})$. The reaction mixture was heated under reflux for $3 \mathrm{~h}$ then poured onto ice/water mixture containing a few drops of hydrochloric acid and the formed solid product was collected by filtration.

\section{2-Amino-5-oxo- $N$-phenyl-4,5,6,7-tetrahydrobenzo[ $b]$ thiophene-3-carboxamide (19a) \\ Pale yellow crystals from ethanol, yield $2.14 \mathrm{~g}$ (75\%), m.p. $120-123{ }^{\circ} \mathrm{C}$. IR (KBr) $v_{\max }\left(\mathrm{cm}^{-1}\right) 3484-3337$ $\left(\mathrm{NH}_{2}, \mathrm{NH}\right), 3055(\mathrm{CH}$, aromatic), $2220(\mathrm{CN}), 1689,1686$ (2 CO), $1630(\mathrm{C}=\mathrm{C}) ;{ }^{1} \mathrm{H}$ NMR (DMSO- $\left.d_{6}, 300 \mathrm{MHz}\right) \delta$ 2.62-2.79 (2t, $\left.4 \mathrm{H}, 2 \mathrm{CH}_{2}\right), 3.02\left(\mathrm{~s}, 2 \mathrm{H}, \mathrm{CH}_{2}\right), 4.75(\mathrm{~s}, 2 \mathrm{H}$, $\mathrm{D}_{2} \mathrm{O}$ exchangeable, $\left.\mathrm{NH}_{2}\right), 7.28-7.40\left(\mathrm{~m}, 5 \mathrm{H}, \mathrm{C}_{6} \mathrm{H}_{5}\right), 8.38$ (s, $1 \mathrm{H}, \mathrm{D}_{2} \mathrm{O}$ exchangeable, $\mathrm{NH}$ ); ${ }^{13} \mathrm{C}$ NMR (DMSO- $d_{6}, 75$ $\mathrm{MHz}) \delta 16.3,36.6,40.3\left(3 \mathrm{CH}_{2}\right), 116.6(\mathrm{CN}), 120.8,122.2$, 125.3, 127.3, 132.2, 134.5, 138.0, $142.9\left(\mathrm{C}_{6} \mathrm{H}_{5}\right.$, thiophene C), 164.3, 179.6 (2CO). Anal. Calcd for $\mathrm{C}_{15} \mathrm{H}_{14} \mathrm{~N}_{2} \mathrm{O}_{2} \mathrm{~S}$ : C, 62.92; H, 4.93; N, 9.78; S, 11.20. Found: C, 63.22; H, 4.75; N, 9.59; S, 11.36. MS: $m / z 286\left(\mathrm{M}^{+}, 42 \%\right)$.}

2-Amino-5-oxo- $N$-( $p$-tolyl)-4,5,6,7-tetrahydrobenzo[b] thiophene-3-carboxamide (19b) 
Yellow crystals from ethanol, yield $1.98 \mathrm{~g}(66 \%)$, m.p. 194-196 ${ }^{\circ} \mathrm{C}$. IR $(\mathrm{KBr}) v_{\max }\left(\mathrm{cm}^{-1}\right) 3449-3327\left(\mathrm{NH}_{2}\right.$, $\mathrm{NH}), 3055(\mathrm{CH}$, aromatic), $2220(\mathrm{CN}), 1689,1687$ (2 $\mathrm{CO}), 1630(\mathrm{C}=\mathrm{C}) ;{ }^{1} \mathrm{H}$ NMR (DMSO- $\left.d_{6}, 300 \mathrm{MHz}\right) \delta$ 2.63-2.77 (2t, $\left.4 \mathrm{H}, 2 \mathrm{CH}_{2}\right), 2.80\left(\mathrm{~s}, 3 \mathrm{H}, \mathrm{CH}_{3}\right), 3.06(\mathrm{~s}, 2 \mathrm{H}$, $\left.\mathrm{CH}_{2}\right), 4.78\left(\mathrm{~s}, 2 \mathrm{H}, \mathrm{D}_{2} \mathrm{O}\right.$ exchangeable, $\left.\mathrm{NH}_{2}\right), 7.26-7.46$ $\left(\mathrm{m}, 4 \mathrm{H}, \mathrm{C}_{6} \mathrm{H}_{4}\right), 8.38\left(\mathrm{~s}, 1 \mathrm{H}, \mathrm{D}_{2} \mathrm{O}\right.$ exchangeable, $\left.\mathrm{NH}\right) ;{ }^{13} \mathrm{C}$ NMR (DMSO- $\left.d_{6}, 75 \mathrm{MHz}\right) \delta 16.3,36.6,40.3\left(3 \mathrm{CH}_{2}\right)$, $116.6(\mathrm{CN}), 120.8,122.2,125.3,127.3,132.7,134.7$, 138.4, $142.6\left(\mathrm{C}_{6} \mathrm{H}_{4}\right.$, thiophene $\left.\mathrm{C}\right), 164.3,179.6$ (2CO). Anal. Calcd for $\mathrm{C}_{16} \mathrm{H}_{16} \mathrm{~N}_{2} \mathrm{O}_{2} \mathrm{~S}$ : C, 63.98; H, 5.37; N, 9.33; S, 10.67. Found: C, 63.72; H, 5.75; N, 9.59; S, 10.36. MS: $\mathrm{m} / \mathrm{z} 300\left(\mathrm{M}^{+}, 36 \%\right)$.

\section{2-Amino- $N$-(4-chlorophenyl)-5-oxo-4,5,6,7- tetrahydrobenzo $[b]$ thiophene-3-carboxamide (19c)}

Brown crystals from 1,4-dioxane, yield $2.49 \mathrm{~g}$ (78\%), m.p. $205-208{ }^{\circ} \mathrm{C}$. IR ( $\left.\mathrm{KBr}\right) v_{\max }\left(\mathrm{cm}^{-1}\right) 3473-$ $3351\left(\mathrm{NH}_{2}, \mathrm{NH}\right), 3055(\mathrm{CH}$, aromatic $), 2220(\mathrm{CN})$, 1689, 1686 (2 CO), $1630(\mathrm{C}=\mathrm{C}) ;{ }^{1} \mathrm{H}$ NMR (DMSO- $d_{6}$, $300 \mathrm{MHz}) \delta 2.64-2.76\left(2 \mathrm{t}, 4 \mathrm{H}, 2 \mathrm{CH}_{2}\right), 3.06(\mathrm{~s}, 2 \mathrm{H}$, $\left.\mathrm{CH}_{2}\right), 4.79$ (s, $2 \mathrm{H}, \mathrm{D}_{2} \mathrm{O}$ exchangeable, $\mathrm{NH}_{2}$ ), 7.23-7.48 $\left(\mathrm{m}, 4 \mathrm{H}, \mathrm{C}_{6} \mathrm{H}_{4}\right), 8.36\left(\mathrm{~s}, 1 \mathrm{H}, \mathrm{D}_{2} \mathrm{O}\right.$ exchangeable, $\left.\mathrm{NH}\right)$; ${ }^{13} \mathrm{C}$ NMR (DMSO- $\left.d_{6}, 75 \mathrm{MHz}\right) \quad \delta 16.1,36.4,40.6$ $\left(3 \mathrm{CH}_{2}\right), 116.9(\mathrm{CN}), 120.2,123.5,125.8,128.6,132.3$, 134.7, 138.0, $142.7\left(\mathrm{C}_{6} \mathrm{H}_{4}\right.$, thiophene $\left.\mathrm{C}\right), 164.3,179.8$ (2CO). Anal. Calcd for $\mathrm{C}_{15} \mathrm{H}_{13} \mathrm{ClN}_{2} \mathrm{O}_{2} \mathrm{~S}: \mathrm{C}, 56.16 ; \mathrm{H}$, 4.08 ; N, 8.73; S, 10.00. Found: C, 56.29; H, 4.26; N, 8.93; S, 10.26. MS: $m / z 320\left(\mathrm{M}^{+}, 28 \%\right)$.

\section{1. 11. General Procedure for the Synthesis of the Tetrahydrobenzo $[b]$ thiophene-3- carboxamide Derivatives 20a-c}

A mixture of either 19a (2.86 g, $0.01 \mathrm{~mol}), 19 \mathrm{~b}(3.00$ $\mathrm{g}, 0.01 \mathrm{~mol})$ or $19 \mathrm{c}(3.20 \mathrm{~g}, 0.01 \mathrm{~mol})$ in $N, N$-dimethylformamide $(30 \mathrm{~mL})$ and ethyl cyanoacetate $(1.07 \mathrm{~g}, 0.01 \mathrm{~mL})$ was heated under reflux for $3 \mathrm{~h}$. The solid product, formed in each case, produced upon pouring onto ice/water mixture, was collected by filtration.

\section{2-(2-Cyanoacetamido)-5-oxo- $N$-phenyl-4,5,6,7-} tetrahydrobenzo $[b]$ thiophene-3-carboxamide (20a)

Dark brown crystals from ethanol, yield $3.28 \mathrm{~g}$ (78\%), m.p 118-120 ${ }^{\circ} \mathrm{C}$. IR (KBr) $v_{\max }\left(\mathrm{cm}^{-1}\right) 3469-3329$ $(\mathrm{NH}), 3054(\mathrm{CH}$, aromatic), $2256(\mathrm{CN}), 1690-1686(3$ CO), $1630(\mathrm{C}=\mathrm{C}) ;{ }^{1} \mathrm{H}$ NMR (DMSO- $\left.d_{6}, 300 \mathrm{MHz}\right) \delta$ 2.61-2.82 (2t, $\left.4 \mathrm{H}, 2 \mathrm{CH}_{2}\right), 3.08\left(\mathrm{~s}, 2 \mathrm{H}, \mathrm{CH}_{2}\right), 3.68(\mathrm{~s}, 2 \mathrm{H}$, $\left.\mathrm{CH}_{2}\right), 7.29-7.42\left(\mathrm{~m}, 5 \mathrm{H}, \mathrm{C}_{6} \mathrm{H}_{5}\right), 8.24,8.34\left(2 \mathrm{~s}, 2 \mathrm{H}, \mathrm{D}_{2} \mathrm{O}\right.$ exchangeable, $2 \mathrm{NH}$ ); ${ }^{13} \mathrm{C}$ NMR (DMSO- $\left.d_{6}, 75 \mathrm{MHz}\right) \delta$ 16.6, 36.2, $40.5\left(3 \mathrm{CH}_{2}\right), 60.3\left(\mathrm{CH}_{2}\right), 170.1(\mathrm{CN}), 120.3$, $123.5,125.9,126.5,132.8,133.3,138.6,142.8\left(\mathrm{C}_{6} \mathrm{H}_{5}\right.$, thiophene C), 164.3, 166.1, 179.6 (3CO). Anal. Calcd for $\mathrm{C}_{18} \mathrm{H}_{15} \mathrm{~N}_{3} \mathrm{O}_{3} \mathrm{~S}: \mathrm{C}, 61.18 ; \mathrm{H}, 4.28 ; \mathrm{N}, 11.89 ; \mathrm{S}, 9.07$. Found: C, 61.31; H, 4.29; N, 11.68; S, 8.85. MS: $m / z 353\left(\mathrm{M}^{+}\right.$, $20 \%)$.
2-(2-Cyanoacetamido)-5-oxo- $N$-phenyl-4,5,6,7tetrahydrobenzo $[b]$ thiophene-3-carboxamide (20b) Brown crystals from ethanol, yield $2.27 \mathrm{~g}(62 \%)$, m.p 211-214 ${ }^{\circ} \mathrm{C}$. IR $(\mathrm{KBr}) v_{\max }\left(\mathrm{cm}^{-1}\right) 3453-3340(\mathrm{NH})$, $3055(\mathrm{CH}$, aromatic), $2256(\mathrm{CN}), 1693-1685$ (3 CO), 1630 $(\mathrm{C}=\mathrm{C}) ;{ }^{1} \mathrm{H}$ NMR (DMSO- $\left.d_{6}, 300 \mathrm{MHz}\right) \delta 2.63-2.80(2 \mathrm{t}$, $\left.4 \mathrm{H}, 2 \mathrm{CH}_{2}\right), 2.70\left(\mathrm{~s}, 3 \mathrm{H}, \mathrm{CH}_{3}\right), 3.10\left(\mathrm{~s}, 2 \mathrm{H}, \mathrm{CH}_{2}\right), 3.62(\mathrm{~s}$, $\left.2 \mathrm{H}, \mathrm{CH}_{2}\right), 7.25-7.38\left(\mathrm{~m}, 4 \mathrm{H}, \mathrm{C}_{6} \mathrm{H}_{4}\right), 8.23,8.38(2 \mathrm{~s}, 2 \mathrm{H}$, $\mathrm{D}_{2} \mathrm{O}$ exchangeable, $2 \mathrm{NH}$ ); ${ }^{13} \mathrm{C}$ NMR (DMSO- $d_{6}, 75 \mathrm{MHz}$ ) $\delta 16.3,36.4,40.8\left(3 \mathrm{CH}_{2}\right), 60.5\left(\mathrm{CH}_{2}\right), 168.3(\mathrm{CN}), 120.6$, $122.1,126.4,127.1,132.8,134.2,138.6,142.9\left(\mathrm{C}_{6} \mathrm{H}_{4}\right.$, thiophene C), 164.2, 166.1, 179.8 (3CO). Anal. Calcd for $\mathrm{C}_{19} \mathrm{H}_{17} \mathrm{~N}_{3} \mathrm{O}_{3} \mathrm{~S}$ : C, 62.11; H, 4.66; N, 11.44; S, 8.73. Found: C, 62.26; H, 4.48; N, 11.52; S, 8.90. MS: $\mathrm{m} / z 367\left(\mathrm{M}^{+}, 28 \%\right)$.

$\mathrm{N}$-(4-Chlorophenyl)-2-(2-cyanoacetamido)-5-oxo4,5,6,7-tetrahydrobenzo $[b]$ thiophene-3-carboxamide (20c)

White crystals from ethanol, yield $2.12 \mathrm{~g}(55 \%)$, m.p $118-121{ }^{\circ} \mathrm{C}$. IR $(\mathrm{KBr}) v_{\max }\left(\mathrm{cm}^{-1}\right) 3470-3338(\mathrm{NH})$, $3055(\mathrm{CH}$, aromatic), $2256(\mathrm{CN}), 1691-1685$ (3 CO), 1630 $(\mathrm{C}=\mathrm{C}) ;{ }^{1} \mathrm{H}$ NMR (DMSO- $\left.d_{6}, 300 \mathrm{MHz}\right) \delta 2.62-2.82(2 \mathrm{t}$, $\left.4 \mathrm{H}, 2 \mathrm{CH}_{2}\right), 3.12\left(\mathrm{~s}, 2 \mathrm{H}, \mathrm{CH}_{2}\right), 3.62\left(\mathrm{~s}, 2 \mathrm{H}, \mathrm{CH}_{2}\right), 7.22-7.49$ $\left(\mathrm{m}, 4 \mathrm{H}, \mathrm{C}_{6} \mathrm{H}_{4}\right), 8.26,8.32\left(2 \mathrm{~s}, 2 \mathrm{H}, \mathrm{D}_{2} \mathrm{O}\right.$ exchangeable, $2 \mathrm{NH}$ ); ${ }^{13} \mathrm{C}$ NMR (DMSO- $\left.d_{6}, 75 \mathrm{MHz}\right) \delta 16.1,36.6,40.4$ $\left(3 \mathrm{CH}_{2}\right), 60.2\left(\mathrm{CH}_{2}\right), 168.9(\mathrm{CN}), 120.4,124.2,125.1,128.6$, $132.5,134.9,138.4,142.6\left(\mathrm{C}_{6} \mathrm{H}_{4}\right.$, thiophene $\left.\mathrm{C}\right), 164.4$, 166.3, 179.6 (3CO). Anal. Calcd for $\mathrm{C}_{18} \mathrm{H}_{14} \mathrm{ClN}_{3} \mathrm{O}_{3} \mathrm{~S}$ : C, 55.74; H, 3.64; N, 10.83; S, 8.27. Found: C, 55.80; H, 3.80; $\mathrm{N}, 11.01 ; \mathrm{S}, 8.38$. MS: $m / z 387\left(\mathrm{M}^{+}, 30 \%\right)$.

\section{1. 12. General Procedure for the Synthesis of the Phenylthioureido Derivatives 21a-c}

Equimolar amounts of either 19a (2.86 g, $0.01 \mathrm{~mol})$, $19 \mathrm{~b}(3.00 \mathrm{~g}, 0.01 \mathrm{~mol})$ or $19 \mathrm{c}(3.20 \mathrm{~g}, 0.01 \mathrm{~mol})$ and phenyl isothiocyanate $(1.30 \mathrm{~g}, 0.01 \mathrm{~mol})$ in ethanol $(40 \mathrm{~mL})$ containing triethylamine $(1.0 \mathrm{~mL})$ were heated under reflux for $3 \mathrm{~h}$ then left to cool. The formed solid crystals, in each case, were collected by filtration.

\section{5-Oxo- $N$-phenyl-2-(3-phenylthioureido)-4,5,6,7- tetrahydrobenzo $[b]$ thiophene-3-carboxamide (21a)}

Yellow crystals from ethanol, yield $2.52 \mathrm{~g}(60 \%)$, m.p. $115-117^{\circ} \mathrm{C}$. IR $(\mathrm{KBr}) v_{\max }\left(\mathrm{cm}^{-1}\right) 3464-3331(\mathrm{NH})$, $3054(\mathrm{CH}$, aromatic), 1689, $1687(2 \mathrm{CO}), 1632(\mathrm{C}=\mathrm{C})$, $1205(\mathrm{C}=\mathrm{S}) ;{ }^{1} \mathrm{H}$ NMR (DMSO- $\left.d_{6}, 300 \mathrm{MHz}\right) \delta 2.63-$ $2.84\left(2 \mathrm{t}, 4 \mathrm{H}, 2 \mathrm{CH}_{2}\right), 3.66\left(\mathrm{~s}, 2 \mathrm{H}, \mathrm{CH}_{2}\right), 7.28-7.40(\mathrm{~m}$, $\left.10 \mathrm{H}, 2 \mathrm{C}_{6} \mathrm{H}_{5}\right), 8.21,8.23,8.38\left(3 \mathrm{~s}, 3 \mathrm{H}, \mathrm{D}_{2} \mathrm{O}\right.$ exchangeable, $3 \mathrm{NH}) ;{ }^{13} \mathrm{C}$ NMR (DMSO- $\left.d_{6}, 75 \mathrm{MHz}\right) \delta 16.1,36.6$, $40.6\left(3 \mathrm{CH}_{2}\right), 120.8,122.4,125.8,126.0,126.3,127.5$, $127.9,128.2,131.9,133.8,139.3,142.9\left(2 \mathrm{C}_{6} \mathrm{H}_{5}\right.$, thiophene C), 164.2, 179.2 (2CO),180.2 (C=S). Anal. Calcd for $\mathrm{C}_{22} \mathrm{H}_{19} \mathrm{~N}_{3} \mathrm{O}_{2} \mathrm{~S}_{2}$ : C, 62.68; H, 4.54; N, 9.97; $\mathrm{S}, 15.21$. Found: C, 62.77; H, 4.49; N, 10.21; S, 15.49. MS: $m / z 421$ $\left(\mathrm{M}^{+}, 32 \%\right)$. 
5-Oxo-2-(3-phenylthioureido)- $N$-(p-tolyl)-4,5,6,7tetrahydrobenzo $[b]$ thiophene-3-carboxamide (21b)

Pale yellow crystals from 1,4-dioxane, yield $2.70 \mathrm{~g}$ (60\%), m.p. $205-208{ }^{\circ} \mathrm{C}$. IR (KBr) $v_{\max }\left(\mathrm{cm}^{-1}\right) 3485-3329$ $(\mathrm{NH}), 3055$ (CH, aromatic), 1689, 1687 (2 CO), 1638 $(\mathrm{C}=\mathrm{C}), 1208(\mathrm{C}=\mathrm{S}) ;{ }^{1} \mathrm{H}$ NMR (DMSO- $\left.d_{6}, 300 \mathrm{MHz}\right) \delta$ 2.61-2.89 (2t, 4H, 2CH 2$), 2.80\left(\mathrm{~s}, 3 \mathrm{H}, \mathrm{CH}_{3}\right), 3.67(\mathrm{~s}, 2 \mathrm{H}$, $\left.\mathrm{CH}_{2}\right), 7.23-7.48\left(\mathrm{~m}, 9 \mathrm{H}, \mathrm{C}_{6} \mathrm{H}_{5}, \mathrm{C}_{6} \mathrm{H}_{4}\right), 8.26,8.21,8.36(3 \mathrm{~s}$, $3 \mathrm{H}, \mathrm{D}_{2} \mathrm{O}$ exchangeable, $3 \mathrm{NH}$ ); ${ }^{13} \mathrm{C}$ NMR (DMSO- $d_{6}, 75$ $\mathrm{MHz}) \delta 16.5,36.6,40.6\left(3 \mathrm{CH}_{2}\right), 30.6\left(\mathrm{CH}_{3}\right), 120.2,121.6$, 124.5, 126.7, 126.8, 128.1, 128.7, 129.3, 133.5, 135.2, 138.6, $142.6\left(\mathrm{C}_{6} \mathrm{H}_{5}, \mathrm{C}_{6} \mathrm{H}_{4}\right.$, thiophene $\left.\mathrm{C}\right), 164.6,179.4$ (2CO), $180.6(\mathrm{C}=\mathrm{S})$. Anal. Calcd for $\mathrm{C}_{23} \mathrm{H}_{21} \mathrm{~N}_{3} \mathrm{O}_{2} \mathrm{~S}_{2}$ : C, 63.42; $\mathrm{H}$, 4.86; N, 9.65; S, 14.72. Found: C, 63.52; H, 4.68; N, 9.74; S, 14.80. MS: $m / z 435\left(\mathrm{M}^{+}, 28 \%\right)$.

\section{$\mathrm{N}$-(4-Chlorophenyl)-5-oxo-2-(3-phenylthioureido)-} 4,5,6,7-tetrahydrobenzo $[b]$ thiophene-3-carboxamide (21c)

Yellow crystals from ethanol, yield $2.95 \mathrm{~g}$ (65\%), m.p. 200-203 ${ }^{\circ} \mathrm{C}$. IR (KBr) $v_{\max }\left(\mathrm{cm}^{-1}\right) 3442-3338(\mathrm{NH}), 3054$ (CH, aromatic), 1689, 1685 (2 CO), $1630(\mathrm{C}=\mathrm{C}), 1208$ $(\mathrm{C}=\mathrm{S}) ;{ }^{1} \mathrm{H}$ NMR (DMSO- $\left.d_{6}, 300 \mathrm{MHz}\right) \delta 2.62-2.86(2 \mathrm{t}$, $\left.4 \mathrm{H}, 2 \mathrm{CH}_{2}\right), 3.68\left(\mathrm{~s}, 2 \mathrm{H}, \mathrm{CH}_{2}\right), 7.22-7.48\left(\mathrm{~m}, 9 \mathrm{H}, \mathrm{C}_{6} \mathrm{H}_{5}\right.$, $\left.\mathrm{C}_{6} \mathrm{H}_{4}\right), 8.22,8.25,8.41$ (3s, $3 \mathrm{H}, \mathrm{D}_{2} \mathrm{O}$ exchangeable, $3 \mathrm{NH}$ ); ${ }^{13} \mathrm{C}$ NMR (DMSO- $\left.d_{6}, 75 \mathrm{MHz}\right) \delta 16.0,36.6,40.9\left(3 \mathrm{CH}_{2}\right)$, 120.3, 124.6, 125.5, 126.6, 127.2, 128.3, 128.7, 128.9, 132.5, 134.7, 138.0, $142.6\left(\mathrm{C}_{6} \mathrm{H}_{5}, \mathrm{C}_{6} \mathrm{H}_{4}\right.$, thiophene $\left.\mathrm{C}\right), 164.5,179.8$ (2CO), $180.5(\mathrm{C}=\mathrm{S})$. Anal. Calcd for $\mathrm{C}_{22} \mathrm{H}_{18} \mathrm{ClN}_{3} \mathrm{O}_{2} \mathrm{~S}_{2}$ : C, 57.95; H, 3.98; N,9.22; S, 14.06. Found: C, 57.63; H, 3.77; N, 9.39; S, 14.26. MS: $m / z 455\left(\mathrm{M}^{+}, 28 \%\right)$.

\section{1. 13. General Procedure for the Synthesis of the Hexahydrobenzo[ $[4,5]$ thieno $[2,3-d]$ pyrimidin-6(5H)-one Derivatives 22a-c}

A suspension of either compound 21a (4.21 g, 0.01 $\mathrm{mol}), 21 \mathrm{~b}$ (4.35 g, $0.01 \mathrm{~mol}), 21 \mathrm{c}(4.55 \mathrm{~g}, 0.01 \mathrm{~mol})$ in sodium ethoxide [prepared through dissolving metallic sodium $(0.46 \mathrm{~g}, 0.02 \mathrm{~mol})$ in absolute ethanol $(50 \mathrm{~mL})]$ was heated in a boiling water bath for $6 \mathrm{~h}$. The reaction mixture was poured onto ice/water mixture then triturated with hydrochloric acid (till pH 7) and the formed solid product was collected by filtration.

3-Phenyl-4-(phenylimino)-2-thioxo-1,2,3,4,7,8hexahydrobenzo $[4,5]$ thieno $[2,3-d]$ pyrimidin- $6(5 H)$ one (22a)

Yellow crystals from ethanol, yield $3.14 \mathrm{~g}$ (78\%), m.p. 111-113 ${ }^{\circ} \mathrm{C}$. IR (KBr) $v_{\max }\left(\mathrm{cm}^{-1}\right) 3458-3324(\mathrm{NH}), 3055$ (CH, aromatic), $1688(\mathrm{CO}), 1632(\mathrm{C}=\mathrm{C}), 1205(\mathrm{C}=\mathrm{S}) ;{ }^{1} \mathrm{H}$ NMR (DMSO- $\left.d_{6}, 300 \mathrm{MHz}\right) \delta 2.61-2.86\left(2 \mathrm{t}, 4 \mathrm{H}, 2 \mathrm{CH}_{2}\right)$, $3.68\left(\mathrm{~s}, 2 \mathrm{H}, \mathrm{CH}_{2}\right), 7.28-7.40\left(\mathrm{~m}, 10 \mathrm{H}, 2 \mathrm{C}_{6} \mathrm{H}_{5}\right), 8.21(\mathrm{~s}, 1 \mathrm{H}$, $\mathrm{D}_{2} \mathrm{O}$ exchangeable, $\left.\mathrm{NH}\right) ;{ }^{13} \mathrm{C}$ NMR (DMSO- $d_{6}, 75 \mathrm{MHz}$ ) $\delta 16.1,36.6,40.6\left(3 \mathrm{CH}_{2}\right), 120.8,122.4,125.8,126.0,126.3$, $127.5,127.9,128.2,132.2,134.7,137.2,142.9\left(2 \mathrm{C}_{6} \mathrm{H}_{5}\right.$, thiophene C), 179.1 (CO), $180.3(\mathrm{C}=\mathrm{S})$. Anal. Calcd for $\mathrm{C}_{22} \mathrm{H}_{17} \mathrm{~N}_{3} \mathrm{OS}_{2}$ : C, 65.48; H, 4.25; N, 10.41; S, 15.89. Found: C, 65.63; H, 4.19; N, 10.32; S, 15.74. MS: $m / z 403\left(\mathrm{M}^{+}\right.$, $38 \%)$.

3-Phenyl-2-thioxo-4-( $p$-tolylimino)-1,2,3,4,7,8hexahydrobenzo $[4,5]$ thieno $[2,3-d]$ pyrimidin- $6(5 H)$ one (22b)

Brown crystals from ethanol, yield $2.46 \mathrm{~g}$ (59\%), m.p. $273-275^{\circ} \mathrm{C}$. IR (KBr) $v_{\max }\left(\mathrm{cm}^{-1}\right) 3479-3336(\mathrm{NH})$, 3055 (CH, aromatic), 1688 (CO), 1631 (C=C), 1209 $(\mathrm{C}=\mathrm{S}) ;{ }^{1} \mathrm{H}$ NMR (DMSO- $\left.d_{6}, 300 \mathrm{MHz}\right) \delta 2.60-2.88(2 \mathrm{t}$, $\left.4 \mathrm{H}, 2 \mathrm{CH}_{2}\right), 2.93\left(\mathrm{~s}, 3 \mathrm{H}, \mathrm{CH}_{3}\right), 3.65\left(\mathrm{~s}, 2 \mathrm{H}, \mathrm{CH}_{2}\right), 7.22-$ $7.45\left(\mathrm{~m}, 9 \mathrm{H}, \mathrm{C}_{6} \mathrm{H}_{5}, \mathrm{C}_{6} \mathrm{H}_{4}\right), 8.25$ (s, $1 \mathrm{H}, \mathrm{D}_{2} \mathrm{O}$ exchangeable, $\mathrm{NH}$ ); ${ }^{13} \mathrm{C}$ NMR (DMSO- $\left.d_{6}, 75 \mathrm{MHz}\right) \delta 16.4,36.8$, $40.9\left(3 \mathrm{CH}_{2}\right), 32.6\left(\mathrm{CH}_{3}\right), 120.2,122.6,125.5,125.9,126.9$, $127.7,127.3,128.1,132.2,134.9,137.6,142.3\left(\mathrm{C}_{6} \mathrm{H}_{5}\right.$, $\mathrm{C}_{6} \mathrm{H}_{4}$, thiophene C), $179.6(\mathrm{CO}), 180.8(\mathrm{C}=\mathrm{S})$. Anal. Calcd for $\mathrm{C}_{23} \mathrm{H}_{19} \mathrm{~N}_{3} \mathrm{OS}_{2}$ : C, 66.16; H, 4.59; N, 10.06; S, 15.36 . Found: C, 66.39; H, 4.42; N, 10.15; S, 15.80. MS: $m / z 417$ $\left(\mathrm{M}^{+}, 42 \%\right)$.

4-((4-Chlorophenyl)imino)-3-phenyl-2-thioxo$1,2,3,4,7,8$-hexahydrobenzo $[4,5]$ thieno $[2,3-d]$ pyrimidin-6 $(5 H)$-one $(22 \mathrm{c})$

Pale yellow crystals from ethanol, yield $3.49 \mathrm{~g}(80 \%)$, m.p. $119-122{ }^{\circ} \mathrm{C}$. IR (KBr) $v_{\max }\left(\mathrm{cm}^{-1}\right) 3459-3328(\mathrm{NH})$, $3054(\mathrm{CH}$, aromatic), 1688, 1685 (2CO), $1630(\mathrm{C}=\mathrm{C})$, $1210(\mathrm{C}=\mathrm{S}) ;{ }^{1} \mathrm{H}$ NMR (DMSO- $\left.d_{6}, 200 \mathrm{MHz}\right) \delta 2.62-2.88$ $\left(2 \mathrm{t}, 4 \mathrm{H}, 2 \mathrm{CH}_{2}\right), 3.68\left(\mathrm{~s}, 2 \mathrm{H}, \mathrm{CH}_{2}\right), 7.22-7.48\left(\mathrm{~m}, 9 \mathrm{H}, \mathrm{C}_{6} \mathrm{H}_{5}\right.$, $\left.\mathrm{C}_{6} \mathrm{H}_{4}\right), 8.22\left(\mathrm{~s}, 1 \mathrm{H}, \mathrm{D}_{2} \mathrm{O}\right.$ exchangeable, $\left.\mathrm{NH}\right) ;{ }^{13} \mathrm{C}$ NMR $\left(\mathrm{DMSO}-d_{6}, 75 \mathrm{MHz}\right) \delta 16.0,36.6,40.9\left(3 \mathrm{CH}_{2}\right), 120.3$, $124.6,125.5,126.6,127.2,128.3,128.7,128.9,132.8,134.3$, 137.2, $142.9\left(\mathrm{C}_{6} \mathrm{H}_{5}, \mathrm{C}_{6} \mathrm{H}_{4}\right.$, thiophene $\left.\mathrm{C}\right), 164.8,179.4$ (2CO), $180.2(\mathrm{C}=\mathrm{S})$. Anal. Calcd for $\mathrm{C}_{22} \mathrm{H}_{16} \mathrm{ClN}_{3} \mathrm{OS}_{2}$ : C, 60.33; H, 3.68; N, 9.59; S, 14.64. Found: C, 60.26; H, 3.49; $\mathrm{N}, 9.41 ; \mathrm{S}, 14.52$. MS: $m / z 437\left(\mathrm{M}^{+}, 32 \%\right)$.

\section{2. Biology Section}

\section{2. 1. Cell Proliferation Assay}

Foretinib was used as the positive control ${ }^{27-29}$ during measuring the anti-proliferative activities of the newly synthesized compounds (Table 1). The newly synthesized compounds were evaluated against the six cancer cell lines A549, HT-29, MKN-45, U87MG, and SMMC-7721 and $\mathrm{H} 460$ using the standard MTT assay in vitro.

The $\mathrm{IC}_{50}$ values were measured through three independent experiments and the data are shown in Table 1. It is clear that many of the tested compounds showed potent anti-proliferative activity with $\mathrm{IC}_{50}$ values less than $6.00 \mu \mathrm{M}$. Generally, the variations of substituents within the aryl moiety together with the heterocyclic ring being attached had a notable effect and a positive impact on the anti-proliferative activity. 
Table 1. In vitro growth inhibitory effects $\mathrm{IC}_{50} \pm \operatorname{SEM}(\mu \mathrm{M})$ of the newly synthesized compounds against cancer cell lines

\begin{tabular}{|c|c|c|c|c|c|c|}
\hline \multirow[t]{2}{*}{ Compound } & \multirow[b]{2}{*}{ A549 } & \multicolumn{4}{|c|}{$\mathrm{IC}_{50} \pm \operatorname{SEM}(\mu \mathrm{M})$} & \multirow[b]{2}{*}{ SMMC-7721 } \\
\hline & & H460 & HT29 & MKN-45 & U87MG & \\
\hline $3 \mathbf{a}$ & $6.62 \pm 2.34$ & $5.42 \pm 2.31$ & $7.73 \pm 2.59$ & $6.72 \pm 2.21$ & $6.82 \pm 1.73$ & $5.01 \pm 2.32$ \\
\hline $3 \mathbf{b}$ & $0.40 \pm 0.25$ & $0.39 \pm 0.17$ & $0.29 \pm 0.16$ & $0.24 \pm 0.16$ & $0.19 \pm 0.17$ & $0.42 \pm 0.23$ \\
\hline $5 \mathbf{a}$ & $4.16 \pm 1.49$ & $4.18 \pm 1.26$ & $5.43 \pm 2.71$ & $5.32 \pm 1.41$ & $6.53 \pm 251$ & $6.80 \pm 1.49$ \\
\hline $5 \mathbf{b}$ & $1.07 \pm 0.68$ & $1.17 \pm 0.63$ & $2.04 \pm 0.95$ & $2.27 \pm 1.33$ & $1.95 \pm 0.49$ & $2.17 \pm 0.52$ \\
\hline $5 c$ & $0.19 \pm 0.07$ & $0.26 \pm 0.11$ & $0.37 \pm 0.21$ & $0.26 \pm 0.08$ & $0.52 \pm 0.23$ & $0.62 \pm 0.25$ \\
\hline $5 d$ & $0.39 \pm 0.15$ & $0.36 \pm 0.21$ & $0.27 \pm 0.18$ & $0.29 \pm 0.17$ & $0.36 \pm 0.22$ & $0.35 \pm 0.18$ \\
\hline $6 \mathbf{a}$ & $6.41 \pm 2.26$ & $5.73 \pm 2.17$ & $6.42 \pm 2.31$ & $8.92 \pm 3.41$ & $6.22 \pm 2.73$ & $5.82 \pm 1.39$ \\
\hline $6 b$ & $1.08 \pm 0.69$ & $0.82 \pm 0.26$ & $0.63 \pm 0.37$ & $0.38 \pm 0.26$ & $1.82 \pm 0.79$ & $0.63 \pm 0.31$ \\
\hline $6 c$ & $0.32 \pm 0.21$ & $0.37 \pm 0.19$ & $0.40 \pm 0.15$ & $0.25 \pm 0.07$ & $0.62 \pm 0.14$ & $0.51 \pm 0.23$ \\
\hline $6 d$ & $0.32 \pm 0.15$ & $0.42 \pm 0.26$ & $0.16 \pm 0.09$ & $0.26 \pm 0.14$ & $0.37 \pm 0.17$ & $0.28 \pm 0.08$ \\
\hline $8 a$ & $0.33 \pm 0.12$ & $0.28 \pm 0.15$ & $0.28 \pm 3.19$ & $6.28 \pm 1.08$ & $7.89 \pm 2.63$ & $9.39 \pm 2.37$ \\
\hline $8 b$ & $0.65 \pm 0.18$ & $0.29 \pm 0.12$ & $0.43 \pm 0.25$ & $0.36 \pm 0.19$ & $0.26 \pm 0.18$ & $0.56 \pm 0.18$ \\
\hline $10 \mathrm{a}$ & $6.48 \pm 1.54$ & $7.60 \pm 2.42$ & $6.63 \pm 2.29$ & $6.16 \pm 2.59$ & $5.26 \pm 1.83$ & $6.29 \pm 2.28$ \\
\hline $10 \mathrm{~b}$ & $2.48 \pm 1.01$ & $3.80 \pm 1.18$ & $2.47 \pm 1.14$ & $4.52 \pm 2.16$ & $0.93 \pm 0.42$ & $2.83 \pm 1.02$ \\
\hline $10 \mathrm{c}$ & $0.26 \pm 0.18$ & $0.43 \pm 0.18$ & $0.41 \pm 0.20$ & $0.38 \pm 0.16$ & $0.46 \pm 0.26$ & $0.38 \pm 0.13$ \\
\hline 10d & $0.65 \pm 0.32$ & $0.58 \pm 0.21$ & $1.08 \pm 0.62$ & $0.73 \pm 0.32$ & $0.46 \pm 0.29$ & $0.53 \pm 0.25$ \\
\hline $12 a$ & $1.82 \pm 0.78$ & $1.06 \pm 0.62$ & $0.84 \pm 0.38$ & $0.61 \pm 0.19$ & $0.72 \pm 0.36$ & $0.59 \pm 0.16$ \\
\hline $12 b$ & $0.22 \pm 0.15$ & $0.36 \pm 0.12$ & $0.43 \pm 0.19$ & $0.29 \pm 0.16$ & $0.42 \pm 0.25$ & $0.58 \pm 0.24$ \\
\hline 14 & $8.68 \pm 2.59$ & $6.70 \pm 2.63$ & $7.28 \pm 1.62$ & $5.63 \pm 1.26$ & $6.92 \pm 2.37$ & $7.29 \pm 2.62$ \\
\hline $16 a$ & $4.91 \pm 1.56$ & $5.41 \pm 1.28$ & $3.52 \pm 1.15$ & $3.30 \pm 1.86$ & $5.02 \pm 2.80$ & $4.69 \pm 1.38$ \\
\hline $16 b$ & $6.53 \pm 2.38$ & $7.24 \pm 2.49$ & $6.80 \pm 1.92$ & $7.49 \pm 2.63$ & $5.60 \pm 1.32$ & $8.05 \pm 3.26$ \\
\hline $16 c$ & $0.76 \pm 0.39$ & $0.68 \pm 0.27$ & $0.43 \pm 0.26$ & $0.51 \pm 0.23$ & $0.60 \pm 0.29$ & $0.39 \pm 0.24$ \\
\hline $17 \mathrm{a}$ & $4.83 \pm 1.53$ & $6.28 \pm 2.54$ & $6.22 \pm 2.26$ & $3.42 \pm 1.60$ & $5.23 \pm 1.21$ & $4.72 \pm 1.38$ \\
\hline $17 b$ & $3.41 \pm 1.25$ & $4.61 \pm 1.28$ & $4.82 \pm 1.52$ & $2.16 \pm 0.73$ & $2.37 \pm 1.29$ & $3.42 \pm 1.53$ \\
\hline $17 \mathrm{c}$ & $3.42 \pm 1.80$ & $2.63 \pm 1.03$ & $4.81 \pm 1.68$ & $3.68 \pm 1.29$ & $3.62 \pm 1.26$ & $2.46 \pm 1.82$ \\
\hline $17 d$ & $1.02 \pm 0.71$ & $1.65 \pm 0.83$ & $0.93 \pm 0.42$ & $0.85 \pm 0.31$ & $0.72 \pm 0.29$ & $0.69 \pm 0.23$ \\
\hline $17 \mathrm{e}$ & $0.82 \pm 0.18$ & $0.51 \pm 0.23$ & $0.42 \pm 0.19$ & $0.31 \pm 0.25$ & $0.39 \pm 0.13$ & $0.58 \pm 0.13$ \\
\hline $17 f$ & $0.21 \pm 0.09$ & $0.33 \pm 0.17$ & $0.48 \pm 0.31$ & $0.35 \pm 0.12$ & $0.59 \pm 0.23$ & $0.23 \pm 0.17$ \\
\hline $19 a$ & $7.28 \pm 2.62$ & $8.29 \pm 2.17$ & $6.39 \pm 1.02$ & $5.86 \pm 2.23$ & $7.43 \pm 2.49$ & $6.32 \pm 2.34$ \\
\hline $19 b$ & $2.34 \pm 1.14$ & $2.28 \pm 0.82$ & $3.51 \pm 1.50$ & $3.46 \pm 1.63$ & $3.68 \pm 1.23$ & $2.18 \pm 1.02$ \\
\hline $19 c$ & $0.24 \pm 0.13$ & $0.32 \pm 0.17$ & $0.48 \pm 0.23$ & $0.29 \pm 0.36$ & $0.26 \pm 0.15$ & $0.43 \pm 0.29$ \\
\hline $20 a$ & $8.52 \pm 3.50$ & $7.62 \pm 2.40$ & $6.39 \pm 3.60$ & $7.80 \pm 2.68$ & $5.18 \pm 2.70$ & $6.48 \pm 2.37$ \\
\hline $20 \mathrm{~b}$ & $4.26 \pm 1.05$ & $5.72 \pm 2.83$ & $6.93 \pm 2.40$ & $4.70 \pm 1.52$ & $5.94 \pm 1.29$ & $1.04 \pm 0.89$ \\
\hline $20 c$ & $0.37 \pm 0.16$ & $0.35 \pm 0.18$ & $0.46 \pm 0.261$ & $0.51 \pm 0.28$ & $0.24 \pm 0.63$ & $0.28 \pm 0.18$ \\
\hline $21 \mathrm{a}$ & $8.24 \pm 3.51$ & $6.39 \pm 2.73$ & $4.46 \pm 1.28$ & $5.34 \pm 1.60$ & $6.70 \pm 2.93$ & $5.45 \pm 1.69$ \\
\hline $21 b$ & $1.42 \pm 0.98$ & $1.64 \pm 0.52$ & $0.68 \pm 0.31$ & $1.25 \pm 0.79$ & $2.35 \pm 1.06$ & $3.36 \pm 1.20$ \\
\hline $21 c$ & $6.43 \pm 2.53$ & $8.69 \pm 2.64$ & $7.16 \pm 1.69$ & $8.69 \pm 2.26$ & $8.53 \pm 2.38$ & $8.76 \pm 2.49$ \\
\hline $22 a$ & $6.42 \pm 2.26$ & $8.25 \pm 2.13$ & $5.29 \pm 2.30$ & $3.27 \pm 1.04$ & $5.52 \pm 1.31$ & $4.50 \pm 1.46$ \\
\hline $22 b$ & $2.43 \pm 1.16$ & $2.37 \pm 1.28$ & $3.45 \pm 1.19$ & $2.26 \pm 1.16$ & $4.58 \pm 1.14$ & $3.27 \pm 3.16$ \\
\hline $22 c$ & $0.42 \pm 0.23$ & $0.29 \pm 0.15$ & $0.54 \pm 0.29$ & $0.19 \pm 0.04$ & $0.59 \pm 0.31$ & $0.42 \pm 0.63$ \\
\hline Foretinib & $0.08 \pm 0.01$ & $0.18 \pm 0.03$ & $0.15 \pm 0.023$ & $0.03 \pm 0.0055$ & $0.90 \pm 0.13$ & $0.44 \pm 0.062$ \\
\hline
\end{tabular}

\section{2. 2. Structure Activity Relationship}

It is clear from Table 1 that most of the compounds exhibited high cyctotoxicities against the six cancer cell lines A549, HT-29, MKN-45, U87MG, and SMMC-7721 and H460. Where in the most of the cases the presence of electronegative substituents is responsible for the high inhibitions. Considering the 2-ylidenecyclohexane-1,3-dione derivatives $\mathbf{3 a}, \mathbf{b}$ it is clear that compound $\mathbf{3 b}(X=S)$ showed higher cytotoxicities than compound 3a $(\mathrm{X}=\mathrm{O})$. For the 4,5,6,7-tetrahydrobenzo $[b]$ thiophene derivatives $\mathbf{5 a}-\mathbf{d}$, compound $\mathbf{5 a}(\mathrm{X}=$ $\mathrm{O}, \mathrm{R}=\mathrm{CN}$ ) exhibited relatively low inhibitions while compound $\mathbf{5 b}(\mathrm{X}=\mathrm{O}, \mathrm{R}=\mathrm{COOEt})$ showed moderate inhibitions. On the other side, compounds $5 c(X=S, R$ $=\mathrm{CN})$ and $\mathbf{5 d}(\mathrm{X}=\mathrm{S}, \mathrm{R}=\mathrm{COOEt})$ showed extensively high inhibitions toward the six cancer cell lines. Similarly, for the 5,6,7,8-tetrahydro- $4 H$-chromene derivatives 6a-d, it is obvious that compound $\mathbf{6 a}(\mathrm{X}=\mathrm{O}, \mathrm{Y}=$ $\left.\mathrm{NH}_{2}\right)$ showed low inhibitions, compound $\mathbf{6 b}(\mathrm{X}=\mathrm{O}, \mathrm{Y}$ $=\mathrm{OH})$ displayed moderate inhibitions and compounds 6c $\left(\mathrm{X}=\mathrm{S}, \mathrm{Y}=\mathrm{NH}_{2}\right)$ and $\mathbf{6 d}\left(\mathrm{X}=\mathrm{S}, \mathrm{Y}=\mathrm{NH}_{2}\right)$ showed high inhibitions. Considering the 2,3,6,7-tetrahydrobenzo $[d]$ thiazole derivatives $\mathbf{8 a}, \mathbf{b}$, where both of the two compounds exhibited high cytotoxicities, this is at- 
tributed to the presence of the thiazole moiety in both compounds. For the 4,5,6,7-tetrahydro- $2 \mathrm{H}$-indazole derivatives 10a-d it is clear that compound 10a $(\mathrm{X}=$ $\mathrm{O}, \mathrm{R}=\mathrm{H}$ ) showed low inhibitions, compound 10b with high inhibition only toward U87MG cell line with $\mathrm{IC}_{50}$ $=0.93 \mu \mathrm{M}$ and moderate inhibitions toward the other five cell lines A549, H460, HT29, MKN-45 and SMMC7721. Compounds 10c $(X=S, R=H)$ and 10d $(X=S$, $\mathrm{R}=\mathrm{Ph}$ ) revealed high inhibitions toward the six cancer cell lines. On the other hand, the 6,7-dihydrobenzo[c] isoxazole derivatives 12a and $\mathbf{1 2 b}$ showed high inhibitions. Compound 14 the 2-(ethoxymethylene)cyclohexane-1,3-dione showed low inhibitions toward the six cancer cell lines. Considering the 2-(arylamino)methylene)cyclohexane-1,3-dione derivatives $16 \mathbf{a}-\mathbf{c}$ where compound 16c $\left(\mathrm{X}=\mathrm{OCH}_{3}\right)$ showed the highest inhibitions among the three compounds although compound 16a $(\mathrm{X}=\mathrm{H})$ showed relatively higher inhibitions than compound 16b $\left(\mathrm{X}=\mathrm{CH}_{3}\right)$. For the 6,7-dihydrobenzo[b]thiophene derivatives $\mathbf{1 7} \mathbf{a}-\mathbf{f}$, it is clear from Table 1 that compounds $17 \mathbf{a}, \mathbf{1 7 b}$ and $17 \mathrm{c}$ showed low inhibitions while compounds 17d ( $\left.\mathrm{X}=\mathrm{CH}_{3}, \mathrm{R}=\mathrm{COOEt}\right)$, 17f $\left(\mathrm{X}=\mathrm{OCH}_{3}, \mathrm{R}=\mathrm{CN}\right)$ and $\mathbf{1 7 d}\left(\mathrm{X}=\mathrm{OCH}_{3}, \mathrm{R}=\right.$ COOEt) showed high inhibitions. Within the 6,7-dihydrobenzo $[b]$ thiophene derivatives $19 \mathbf{a}-\mathbf{c}$ and $20 \mathbf{a}-\mathbf{c}$, compounds 19c $(\mathrm{Y}=\mathrm{Cl})$ and 20c $(\mathrm{X}=\mathrm{Cl})$ showed the highest inhibitions among the six compounds. Surprisingly, for compounds $21 \mathbf{a}-\mathbf{c}$ where compound $\mathbf{2 1 b}(\mathrm{Y}=$ $\left.\mathrm{CH}_{3}\right)$ showed higher inhibitions than 21a $(\mathrm{Y}=\mathrm{H})$ and 21c $(\mathrm{Y}=\mathrm{Cl})$. Finally for the $1,2,3,4,7,8$-hexahydrobenzo[4,5]thien [2,3-d]pyrimidine derivatives $22 \mathbf{a}-\mathbf{c}$, where compound 22a $(\mathrm{Y}=\mathrm{H})$ showed low inhibitions, compound 22b $\left(\mathrm{Y}=\mathrm{CH}_{3}\right)$ displayed moderate inhibitions and compound 22c $(\mathrm{Y}=\mathrm{Cl})$ showed high inhibitions. It is of great value to mention that compounds $\mathbf{3 b}, \mathbf{5 c}, \mathbf{5 d}$, 6b, 6c, 6d, 8a, 8b, 10c, 10d, 12a, 12b, 16c, 17d, 17e, 17f, 19c, 20c and 22c were the most cytotoxic compounds among the tested compounds.

\section{2. 3. Inhibition of Tyrosine Kinases}

Compounds $3 \mathrm{~b}, \mathbf{5 c}, \mathbf{5 d}, \mathbf{6 b}, \mathbf{6 c}, \mathbf{6 d}, \mathbf{8 a}, \mathbf{8 b}, \mathbf{1 0 c}, \mathbf{1 0 d}$, 12a, 12b, 16c, 17d, 17e, 17f, 19c, 20c and 22c were selected for inhibition of the five tyrosine kinases c-Kit, Flt-3, VEGFR-2, EGFR, and PDGFR. It is clear from Table 2 that compounds $\mathbf{3 b}, \mathbf{5 d}, \mathbf{6 b}, \mathbf{6 d}, \mathbf{8 a}, \mathbf{8 b}, \mathbf{1 0 c}, \mathbf{1 2 a}$, $12 b, 16 c, 17 e, 17 f$ and $19 c$ were the most potent of the tested compounds towards the five tyrosine kinases. Compound $\mathbf{5 c}$ showed high potency towards the two kinases c-EGFR and PDGFR with $\mathrm{IC}_{50} 0.96$ and $0.68 \mathrm{nM}$, respectively. In addition the 2-((4-methoxyphenyl)amino)methylene)cyclohexane-1,3-dione (16c) showed activity towards the five kinases with $\mathrm{IC}_{50} 0.14,0.32,0.21$, 0.36 and $0.40 \mathrm{nM}$, respectively. Compounds $6 \mathrm{c}, \mathbf{1 0 d}, 20 \mathrm{c}$ and 22c showed the lowest potency among the tested compounds.
Table 2. Inhibition of tyrosine kinases (enzyme $\mathrm{IC}_{50}$ in $\mathrm{nM}$ ) by compounds $3 \mathrm{~b}, 5 \mathrm{c}, 5 \mathrm{~d}, 6 \mathrm{~b}, 6 \mathrm{c}, 6 \mathrm{~d}, 8 \mathrm{a}, 8 \mathrm{~b}, 10 \mathrm{c}, 10 \mathrm{~d}, 12 \mathrm{a}, 12 \mathrm{~b}, 16 \mathrm{c}, 17 \mathrm{~d}$, $17 \mathrm{e}, 17 \mathrm{f}, 19 \mathrm{c}, 20 \mathrm{c}$ and $22 \mathrm{c}$

\begin{tabular}{lccccc}
\hline Compound & c-Kit & Flt-3 & VEGFR-2 & EGFR & PDGFR \\
\hline 3b & 0.80 & 0.37 & 0.42 & 0.58 & 0.38 \\
5c & 1.03 & 2.63 & 1.82 & 0.96 & 0.68 \\
5d & 0.23 & 0.26 & 0.42 & 0.69 & 0.72 \\
6b & 0.48 & 0.27 & 0.62 & 0.49 & 0.52 \\
6c & 1.42 & 2.58 & 1.61 & 1.80 & 2.31 \\
6d & 0.16 & 0.24 & 0.57 & 0.34 & 0.28 \\
8a & 0.58 & 0.42 & 0.38 & 0.27 & 0.19 \\
8b & 0.29 & 0.48 & 0.68 & 0.52 & 0.40 \\
10c & 0.16 & 0.13 & 0.28 & 0.31 & 0.28 \\
10d & 2.07 & 1.24 & 1.30 & 1.28 & 1.72 \\
12a & 0.36 & 0.24 & 0.62 & 0.18 & 0.24 \\
12b & 0.18 & 0.53 & 0.61 & 0.53 & 0.42 \\
16c & 0.14 & 0.32 & 0.21 & 0.36 & 0.40 \\
17d & 1.85 & 1.64 & 1.52 & 2.83 & 1.18 \\
17e & 0.26 & 0.23 & 0.37 & 0.28 & 0.46 \\
17f & 0.55 & 0.80 & 0.92 & 0.16 & 0.27 \\
19c & 0.26 & 0.42 & 0.31 & 0.50 & 0.62 \\
20c & 1.27 & 1.43 & 2.60 & 2.88 & 1.69 \\
22c & 2.49 & 2.61 & 1.96 & 2.37 & 3.39 \\
\hline
\end{tabular}

\section{2. 4. Inhibition of Selected Compounds Towards Pim-1 Kinase}

Compounds 3b, 5d, 6b, 6d, 8a, 8b, 10c, 12a, 12b, $16 \mathrm{c}, 17 \mathrm{e}, 17 \mathrm{f}$ and $19 \mathrm{c}$ were selected to examine their Pim1 kinase inhibition activity (Table 3 ) as these compounds showed high inhibition towards the tested cancer cell lines at a range of 10 concentrations and the $\mathrm{IC}_{50}$ values were calculated. Compounds 5d, 6b, 6d, 10c, 12a, 17e and $\mathbf{1 7 f}$ were the most potent to inhibit Pim-1 activity with $\mathrm{IC}_{50}$ values of $0.24,0.41,0.30,0.28,0.45,0.23$ and $0.25 \mu \mathrm{M}$, respectively. On the other hand, compounds $3 \mathbf{b}$, 8a, 8b, 12b, 16c, and 19c were less effective $\left(\mathrm{IC}_{50}>10\right.$ $\mu \mathrm{M})$. SGI-1776 was used as the positive control with $\mathrm{IC}_{50}$ $0.048 \mu \mathrm{M}$ in the assay. These profiles in combination with cell growth inhibition data of compounds $\mathbf{3 b}, \mathbf{5 d}, \mathbf{6 b}, \mathbf{6 d}$, $8 \mathrm{a}, 8 \mathrm{~b}, 10 \mathrm{c}, 12 \mathrm{a}, 12 \mathrm{~b}, 16 \mathrm{c}, 17 \mathrm{e}, 17 \mathrm{f}$ and $19 \mathrm{c}$ are listed in Table 3 indicating that Pim- 1 is a potential target of these compounds.

\section{Results and Discussion}

The synthesis of the 2-(hetero-2-ylmethylene)cyclohexane-1,3-dione derivatives $\mathbf{3 a}, \mathbf{b}$ has been accomplished as outlined in Scheme 1 starting from cyclohexan-1,3-dione (1). Compounds $\mathbf{3} \mathbf{a}$ and $\mathbf{3} \mathbf{b}$ were obtained through the reaction of $\mathbf{1}$ with either of furan-2-carbaldehyde or thiophene-2-carbaldehyde. The reaction of either of compound $\mathbf{3} \mathbf{a}$ or $\mathbf{3 b}$ with elemental sulfur and either of malononitrile (4a) or ethyl cyanoacetate $(\mathbf{4} \mathbf{b})$ gave the 6,7-dihydrobenzo $[b]$ thiophen-5(4H)-one derivatives $\mathbf{5 a -}$ 
Table 3. The inhibitory activity of compounds $3 \mathbf{b}, \mathbf{5 d}, \mathbf{6 b}, \mathbf{6 d}, \mathbf{8 a}, \mathbf{8 b}$, 10c, 12a, 12b, 16c, 17e, 17f and 19c toward Pim-1 kinase.

\begin{tabular}{lcc}
\hline Compound & $\begin{array}{c}\text { Inhibition ratio } \\
\text { at } \mathbf{1 0} \boldsymbol{\mu M}\end{array}$ & $\begin{array}{c}\mathbf{I C}_{\mathbf{5 0}} \\
(\boldsymbol{\mu M})\end{array}$ \\
\hline 3b & 16 & $>10$ \\
$\mathbf{5 d}$ & 96 & 0.24 \\
$\mathbf{6 b}$ & 90 & 0.41 \\
$\mathbf{6 d}$ & 89 & 0.30 \\
$\mathbf{8 a}$ & 26 & $>10$ \\
$\mathbf{8 b}$ & 24 & $>10$ \\
$\mathbf{1 0 c}$ & 92 & 0.28 \\
$\mathbf{1 2 a}$ & 0.88 & 0.45 \\
$\mathbf{1 2 b}$ & 28 & $>10$ \\
$\mathbf{1 6 c}$ & 26 & $>10$ \\
$\mathbf{1 7}$ & 92 & 0.23 \\
$\mathbf{1 7 f}$ & 90 & 0.25 \\
$\mathbf{1 9 c}$ & 18 & $>10$ \\
SGI-1776 & - & 0.048 \\
\hline
\end{tabular}

d, respectively. The structures of the latter products were established on the analytical and spectral data. Thus, the ${ }^{1} \mathrm{H}$ NMR spectrum of compound 5a (as an example) showed the presence of two triplets at $\delta 2.62-2.78 \mathrm{ppm}$ for the two $\mathrm{CH}_{2}$ groups, a singlet at $\delta 4.73 \mathrm{ppm}\left(\mathrm{D}_{2} \mathrm{O}\right.$ exchangeable $)$ indicating the presence of the $\mathrm{NH}_{2}$ group, a singlet at $\delta$ 6.84 for the pyran $H-4$ and a multiplet at $\delta 6.82-7.86 \mathrm{ppm}$ for the furan protons. In addition, the ${ }^{13} \mathrm{C}$ NMR spectrum revealed three signals at $\delta 16.6,36.3$ and 39.5 equivalent to the three $\mathrm{CH}_{2}$ groups, two signals at $\delta 112.6$ and 158.4 for the $\mathrm{C}=\mathrm{CH}$ group, a signal at $\delta 116.8$ for the $\mathrm{CN}$ group, eight signals at $\delta 135.4,140.6,141.4,142.2,142.7,144.8$, 145.6, 146.5 for the thiophene and furan carbons and a signal at $\delta 179.3$ indicating the $\mathrm{CO}$ group. The reaction of either compound $\mathbf{3} \mathbf{a}$ or $\mathbf{3} \mathbf{b}$ with either of malononitrle (4a) or ethyl cyanoacetate $(\mathbf{4 b})$ in ethanol containing a catalytic amount of triethylamine gave the $2 \mathrm{H}$-chromen-5-one derivatives $\mathbf{6 a}-\mathbf{d}$, respectively (Scheme 1 ).<smiles>O=C1CCCC(=O)C1</smiles><smiles>OCc1[X]ccc1</smiles>

1<smiles>O=C1CCCC(=O)C1=Cc1ccco1</smiles>

$$
\begin{array}{r}
\text { 2a, } X=O \\
\text { b, } X=S
\end{array}
$$

$$
\begin{array}{r}
\text { 3a, } X=O \\
\text { b, } X=S
\end{array}
$$

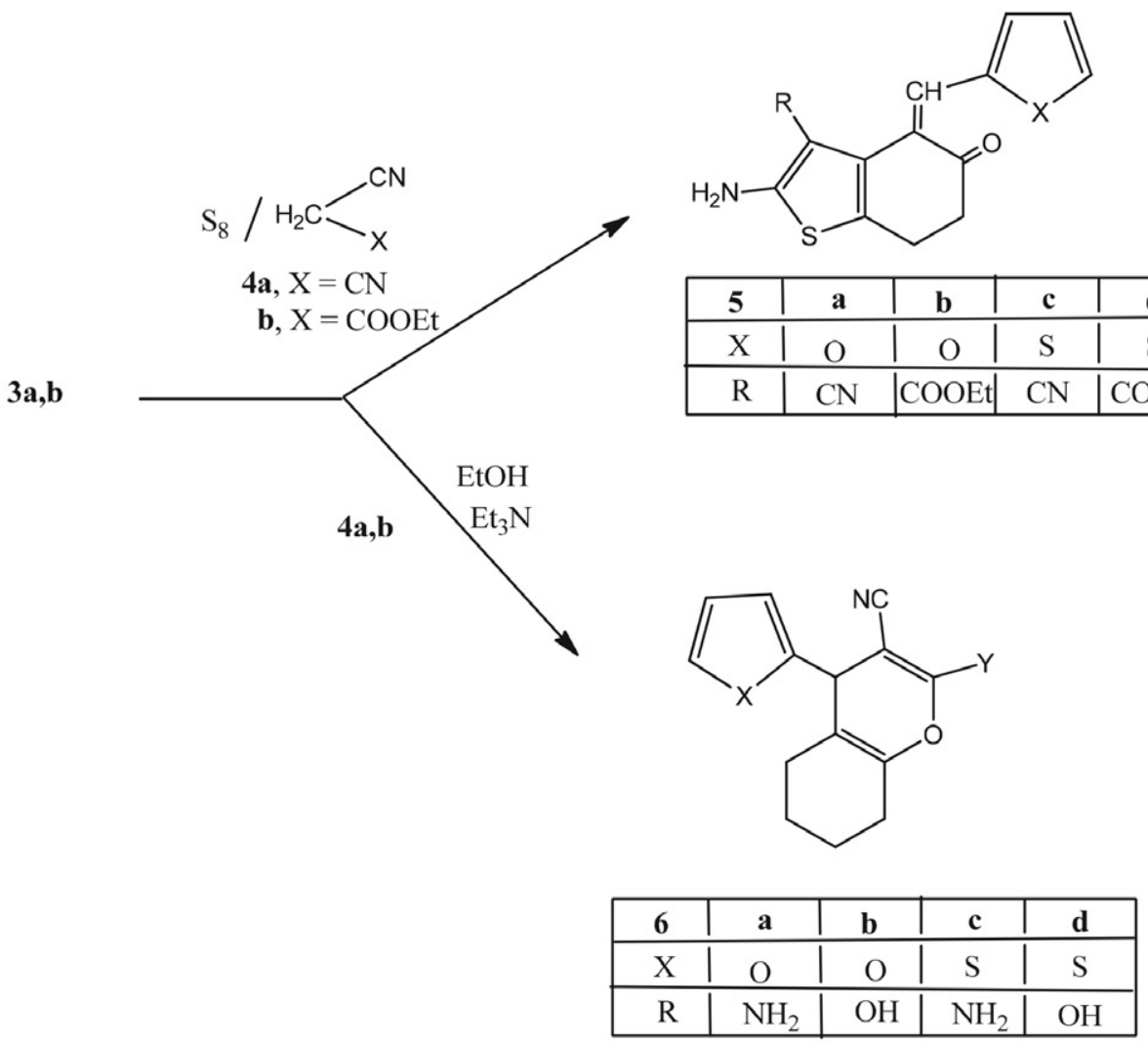

Scheme 1. Synthesis of compounds 3a,b; 5a-d and 6a-d. 
The reactivity of compounds $\mathbf{3} \mathbf{a}$ and $\mathbf{3} \mathbf{b}$ toward thiazole synthesis was studied. Thus, the reaction of either compound $\mathbf{3 a}$ or $\mathbf{3 b}$ with elemental sulfur and phenyl isothiocyanate (7) gave the 2-thioxohexahydrobenzo[d]thiazole derivatives $\mathbf{8 a}$ and $\mathbf{8 b}$, respectively.

The reaction of either of compound $\mathbf{3 a}$ or $\mathbf{3} \mathbf{b}$ with either hydrazine hydrate $(\mathbf{9 a})$ or phenylhydrazine $(\mathbf{9 b})$ gave the 4-hydrazono-4,5,6,7-tetrahydro- $2 \mathrm{H}$-indazole derivatives $10 a-d$, respectively. Similarly, the reaction of either 3a or $\mathbf{3 b}$ with hydroxylamine hydrochloride (11) gave the 6,7-dihydrobenzo $[c]$ isoxazol-4 $(5 H)$-one oxime derivatives 12a and $\mathbf{1 2 b}$, respectively (Scheme 2).

Next, we moved toward studying the use of 2-(ethoxymethylene)cyclohexane-1,3-dione (14), obtained according to the reported work ${ }^{30}$ via the reaction of cyclohexane-1,3-dione (1) with ethyl orthoformate in acetic acid solution, through different heterocyclization reactions.
Thus, the reaction of compound 3 with any of the aromatic amines namely aniline (15a), 4-methylaniline (15b) or 4-methoxyaniline (15c) gave the 2 -(aminomethylene) cyclohexane-1,3-dione derivatives $\mathbf{1 6 a}-\mathbf{c}$, respectively. Structures of compounds $\mathbf{1 6 a - c}$ were confirmed on the basis of their respective analytical and spectral data (see experimental section). Compounds $\mathbf{1 6} \mathbf{a}-\mathbf{c}$ were used to synthesize thiophene derivatives using the Gewald's thiophene synthesis. ${ }^{31-33}$ Thus, the reaction of any of compounds 16a, 16b or 16c with elemental sulfur and either of malononitrile (4a) or ethyl cyanoacetate $(\mathbf{4 b})$ gave the 6,7-dihydrobenzo[b]thiophene derivatives $\mathbf{1 7 a - f}$, respectively.

The reaction of cycohexane-1,3-dione (1) with elemental sulfur and any of cyanoacetanilide (19a), cyano-4-methylacetanilide (19b) or cyano-4-methoxyacetanilide (19c) gave the 6,7-dihydrobenzo[b]thiophene

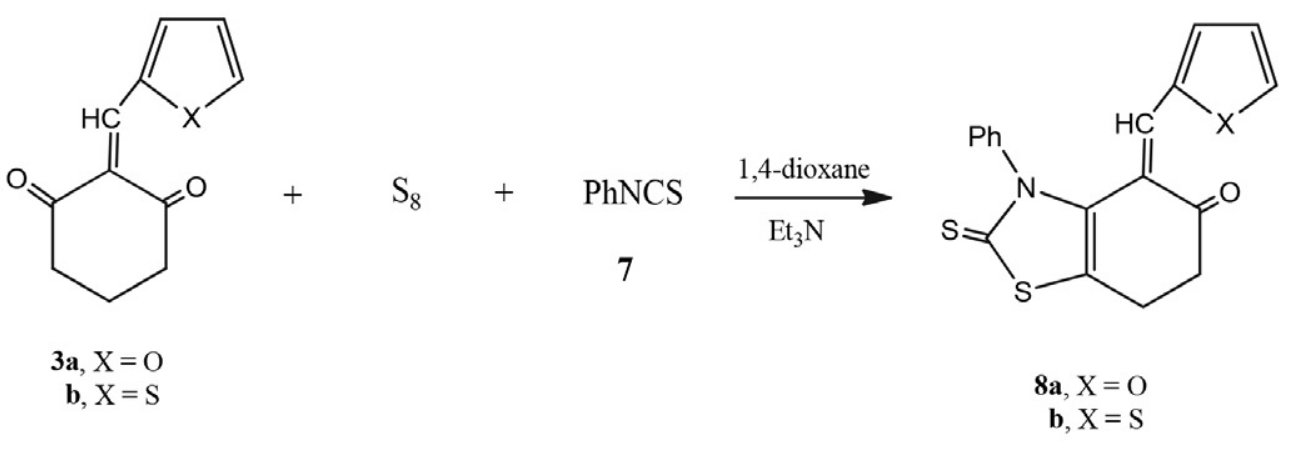<smiles></smiles>

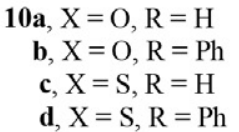<smiles>[X]c1ccccc1-c1onc2c1C(=N)CCC2</smiles> 

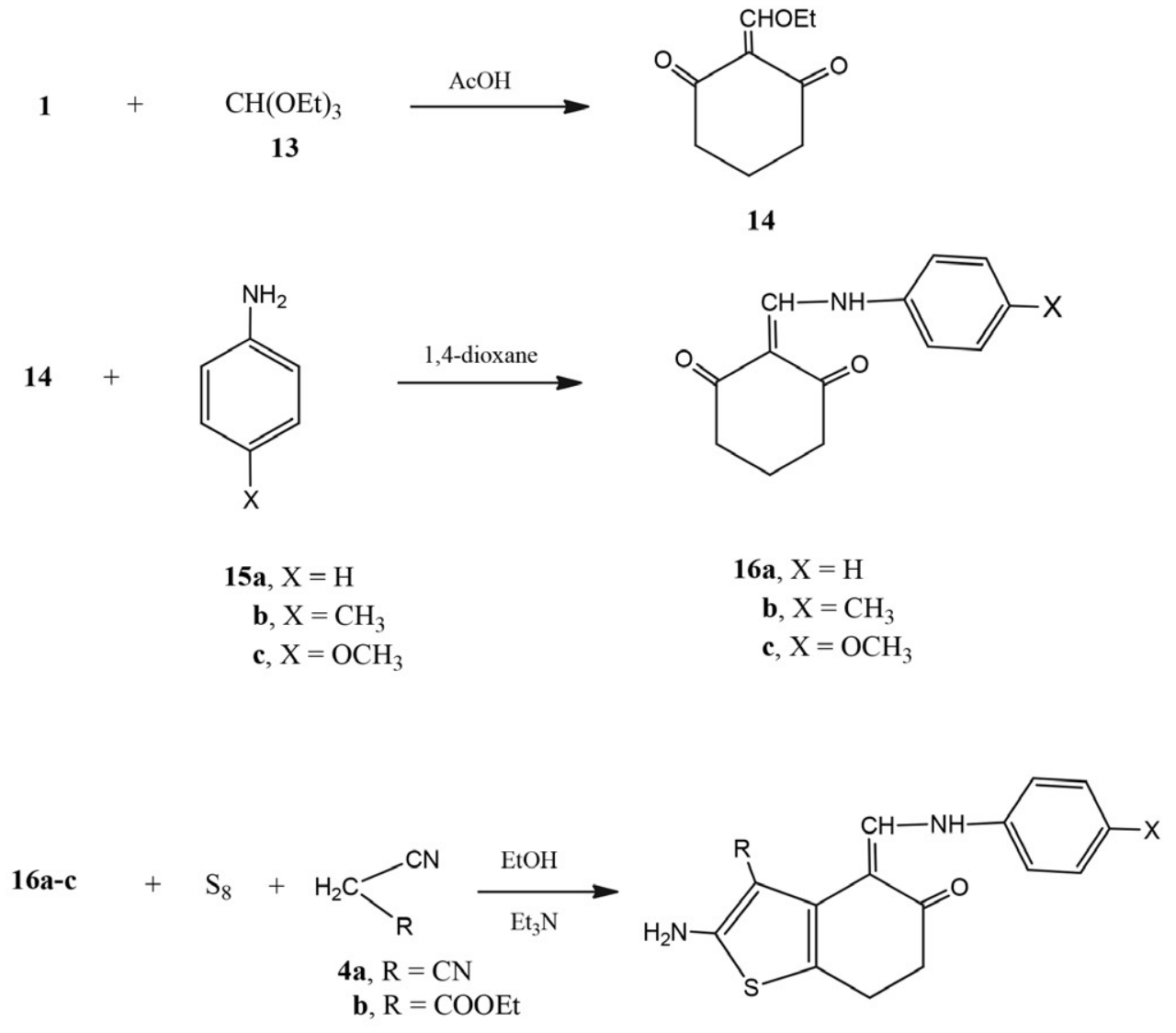

\begin{tabular}{|c|c|c|c|c|c|c|}
\hline 17 & a & b & c & d & e & f \\
\hline$X$ & $\mathrm{H}$ & $\mathrm{H}$ & $\mathrm{CH}_{3}$ & $\mathrm{CH}_{3}$ & $\mathrm{OCH}_{3}$ & $\mathrm{OCH}_{3}$ \\
\hline $\mathrm{R}$ & $\mathrm{CN}$ & COOEt & $\mathrm{CN}$ & COOEt & $\mathrm{CN}$ & COOEt \\
\hline
\end{tabular}

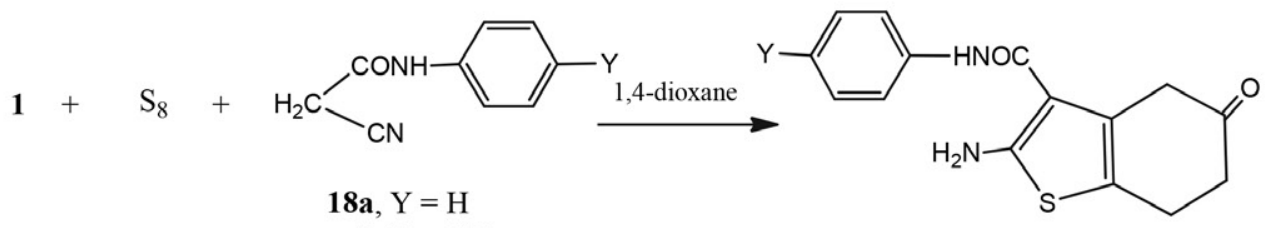

b, $\mathrm{Y}=\mathrm{CH}_{3}$

c, $\mathrm{Y}=\mathrm{Cl}$

19a, $\mathrm{Y}=\mathrm{H}$

b, $\mathrm{Y}=\mathrm{CH}_{3}$

c, $\mathrm{Y}=\mathrm{Cl}$

Scheme 3. Synthesis of compounds 14, 16a-c; 17a-f and 19a-c.

derivatives 19a-c, respectively (Scheme 3). The analytical and spectral data of the latter compounds were consistent with their respective structures. Thus, the ${ }^{1} \mathrm{H}$ NMR of compound 19a showed the presence of two triplets at $\delta$ 2.62-2.79 ppm equivalent to the two $\mathrm{CH}_{2}$ groups, a singlet for the third $\mathrm{CH}_{2}$ group, a singlet at $\delta 4.75 \mathrm{ppm}\left(\mathrm{D}_{2} \mathrm{O}\right.$ exchangeable) for the $\mathrm{NH}_{2}$ group, a multiplet at $\delta 7.28-7.40$ ppm for the phenyl protons and a singlet at $\delta 8.38 \mathrm{ppm}$ $\left(\mathrm{D}_{2} \mathrm{O}\right.$ exchangeable) indicating the $\mathrm{NH}$ group. Moreover, the ${ }^{13} \mathrm{C}$ NMR spectrum showed the presence of three sig- nals at $\delta 16.3,36.6,40.3$ corresponding to the three $\mathrm{CH}_{2}$ groups, a signal at $\delta 116.6$ indicating the presence of the $\mathrm{CN}$ group, signals at $\delta 120.8,122.2,125.3,127.3,132.2$, $134.5,138.0,142.9$ for the $\mathrm{C}_{6} \mathrm{H}_{5}$ and thiophene carbons and two signals at $\delta 164.3,179.6$ confirming the presence of two CO groups.

Compounds 19a-c were ready to form amide derivatives through their reactions with cyanomethylene esters. Thus, the reaction of either compounds $19 \mathbf{a}-\mathbf{c}$ with ethyl cyanoacetate in $\mathrm{N}, \mathrm{N}$-dimethylformamide solution 
Scheme 4. Synthesis of compounds 20a-c; 21a-c and 22a-c.

took place to form the 4,5,6,7-tetrahydrobenzo[b]thiophen-2-yl)acetamide derivatives $\mathbf{2 0 a}-\mathbf{c}$, respectively. On the other hand, the reaction of either compounds 19a, 19b or $9 \mathbf{c}$ with phenyl isothiocyanate gave the corresponding $\mathrm{N}$-phenylthiourea derivatives $\mathbf{2 1 a - c}$, respectively. The analytical and spectral data of compounds 21 a-c were consistent with their respective structures. Compounds 20a-c were ready for further cyclization to form biologically active annulated compounds. Thus, heating of either compound $21 \mathrm{a}, 21 \mathrm{~b}$ or $21 \mathrm{c}$ in sodium ethoxide solution in a boiling water bath afforded the hexahydrobenzo[4,5] thieno[2,3-d]pyrimidine derivatives 22a-c, respectively (Scheme 4). Their structures were based on the analytical and spectral data (see experimental section).

\section{Conclusion}

Forty novel heterocyclic compounds bearing cyclohexanone moiety were designed and synthesized. Their structures were confirmed by multiple techniques. The synthesized compounds were screened for cytotoxic activity against a panel of six human cancer cell lines using MTT assay. Some intriguing structure-activity relationships were found and discussed and the most active compounds were selected for further screening against tyrosine kinases, Pim-1 kinase and the results indicated that these compounds are good condidates as anti-cancer agents that will encourange further work in the future.

\section{Human and Animal Rights}

No Animals/Humans were used for studies that are basis of this research.<smiles>[Y]c1ccc(NC(=O)c2c(NC(=O)CN)sc3c2CC(=O)CC3)cc1</smiles>

$$
\begin{aligned}
\text { 20a, } \mathrm{Y} & =\mathrm{H} \\
\text { b, } \mathrm{Y} & =\mathrm{CH}_{3} \\
\text { c, } \mathrm{Y} & =\mathrm{Cl}
\end{aligned}
$$<smiles>[Y]c1ccc(NC(=O)c2c(NC(N)=S)sc3c2CC(=O)CC3)cc1</smiles>

21a, $\mathrm{Y}=\mathrm{H}$

b, $\mathrm{Y}=\mathrm{CH}_{3}$

c, $\mathrm{Y}=\mathrm{Cl}$<smiles>[Y]c1ccc(/N=c2/c3c4c(sc3[nH]c(=S)n2-c2ccccc2)CCC(=O)C4)cc1</smiles>

22a, $\mathrm{Y}=\mathrm{H}$

b, $\mathrm{Y}=\mathrm{CH}_{3}$

c, $\mathrm{Y}=\mathrm{Cl}$

\section{Consent for Publication}

This work is consent for publication through the Journal formats.

\section{Conflict of Interest}

The authors declare no conflict of interest, financial or otherwise.

\section{References}

1. W. Pfau, H. Marquardt, Toxicology 2001, 166, 25-30.

DOI:10.1016/S0300-483X(01)00435-8 
2. V. P. Boyarskiy, K. V. Luzyanin, V. Y. Kukushkin, Coord. Chem. Rev. 2012, 256, 2029-2056. DOI:10.1016/j.ccr.2012.04.022

3. R. K. Singh, N. Sinha, S. Jain, M. Salman, F. Naqvi, N. Anand, Tetrahedron 2005, 61, 8868-8874.

DOI:10.1016/j.tet.2005.07.018

4. V. Frenna, G. Macaluso, G. Consiglio, B. Cosimelli, D. Spinelli, Tetrahedron 1999, 55, 12885-12896.

DOI:10.1016/S0040-4020(99)00762-0

5. B. S. Jursic, F. Douelle, K. Bowdy, E. D. Stevens, Tetrahedron Lett. 2002, 43, 5361-5365.

DOI:10.1016/S0040-4039(02)00799-2

6. K. V. Padoley, S. N. Mudliar, R. A. Pandey, Bioresour. Technol. 2008, 99, 4029-4043. DOI:10.1016/j.biortech.2007.01.047

7. A. W. Erian, S. M. Sherif, A. A. Alassar, Y. M. Elkholy, Tetrahedron 1994, 50,1877-1884.

DOI:10.1016/S0040-4020(01)80859-0

8. J. Bergman, S. Bergman, T. Brimert, Tetrahedron 1999, 55, 10447-10466. DOI:10.1016/S0040-4020(99)00570-0

9. J. Fuentes, W. Moreda, C. Ortiz, I. Robina, C. Welsh, Tetrahedron 1992, 48, 6413-6424.

DOI:10.1016/S0040-4020(01)88231-4

10. S. Buscemi, A. Pace, I. Pibiri, N. Vivona, T. Caronna, J. Fluor. Chem. 2004, 125, 165-173.

DOI:10.1016/j.jfluchem.2003.07.006

11. M. R. Shaaban, T. S. Saleh, A. S. Mayhoub, A. Mansour, A. M. Farag, Bioorg. Med. Chem. 2008, 16, 6344-6352.

DOI:10.1016/j.bmc.2008.05.011

12. M. G. Rimoli, L. Avallone, P. de Caprariis, E. Luraschi, E. Abignente, W. Filippelli, L. Berrino, F. Rossi, Eur. J. Med. Chem. 1997, 32, 195-203. DOI:10.1016/S0223-5234(97)83971-2

13. L. Feng, K. W. Yang, L. S. Zhou, J. M. Xiao, X. Yang, L. Zhai, Y. L. Zhang, M. W. Crowder, Bioorg. Med. Chem. Lett. 2012, 22, 5185-5189. DOI:10.1016/j.bmcl.2012.06.074

14. S. Günal, N. Kaloğlu, S. I. Özdemir, I. D. Özdemir, Inorg. Chem. Comm. 2012, 21, 142-146.

DOI:10.1016/j.inoche.2012.04.033

15. S. K. Srivastava, W. Haq, P. M. S. Chauhan, Bioorg. Med. Chem. Lett. 1999, 9, 965-966.

DOI:10.1016/S0960-894X(99)00128-6

16. D. Z. Li,Y. Li, X. G. Chen, C. G. Zhu, J. Yang, H. Y. Liu, X. D. Pan, Chin. Chem. Lett. 2007, 18, 1335-1338.

DOI:10.1016/j.cclet.2007.09.014

17. Z. Li, Q. Yang, X. Qian, Bioorg. Med. Chem. 2005, 13, 31493155. DOI:10.1016/j.bmc.2005.02.045

18. R. J. Pagliero, S. Lusvarghi, A. B. Pierini, R. Brun, M. R. Mazzieri, Bioorg. Med. Chem. 2010, 18, 142-150.

DOI:10.1016/j.bmc.2009.11.010
19. S. Barbuceanu, G. Saramet, G. L. Almajan, C. Draghici, F. Barbuceanu, G. Bancescu, Eur. J. Med. Chem. 2012, 49, 417-423. DOI:10.1016/j.ejmech.2012.01.031

20. A. Da Settimo, G. Primofiore, F. Da Settimo, L. Calzolari, P. Cazzulani, A. Passoni, O. Tofanetti, Eur. J. Med Chem. 1992, 27,395-400. DOI:10.1016/0223-5234(92)90153-R

21. A. E. Amr, M. H. Sherif, M. G. Assy, M. A. Al-Omar, I. Ragab, Eur. J. Med. Chem. 2010, 45, 5935-5942.

DOI:10.1016/j.ejmech.2010.09.059

22. T. Bányász, J. Magyar, A. Varró, A. Kovács, I. Gyönös, G. Szénási, P. Nánási, Vasc. Pharmacol. 1999, 32, 329-333. DOI:10.1016/S0306-3623(98)00197-9

23. D. M. Swanson, C. R. Shah, B. Lord, K. Morton, L. K. Dvorak, C. Mazur, R. Apodaca, W. Xiao, J. D. Boggs, M. Feinstein, S. Wilson, A. J. Barbier, P. Bonaventure, T. W. Lovenberg, N. I. Carruthers, Eur. J. Med. Chem. 2009, 44, 4413-4425.

DOI:10.1016/j.ejmech.2009.06.007

24. R. M. Mohareb, R. A. Ibrahim, E. S. Alwan, Acta Chim. Slov. 2020, in press. DOI:10.17344/acsi.2020.6090

25. R. M. Mohareb, F. M. Manhi, A. Abdelwahab, Acta Chim. Slov. 2020, 67, 83-95. DOI:10.17344/acsi.2019.5224

26. R. M. Mohareb, P. A. Halim, Acta Chim. Slov. 2018, 65, 554568. DOI:10.17344/acsi.2017.4146

27. L. Liu, A. Siegmund, N. Xi, P. Kaplan-Lefko, K. Rex, A. Chen. J. Lin, J. Moriguchi, L. Berry, L. Y. Huang, Y. Teffera, Y. J. Yang, Y. H. Zhang, S. F. Bellon, M. Lee, R. Shimanovich, A. Bak, C. Dominguez, M. H. Norman, J. C. Harmange, I. Dussault, T. S. Kim, J. Med. Chem. 2008, 51, 3688-3691.

DOI: $10.1021 /$ jm800401t

28. M. L. Peach, N. Tan, S. J. Choyke, A. Giubellino, G. Athauda, T. R. Burke, M. C. Nicklaus, D. P. Bottaro, J. Med. Chem. 2009, 52, 943-951. DOI:10.1021/jm800791f

29. F. D. Bacco, P. Luraghi, E. Medico, G. Reato, F. Girolami, T. Perera, P. Gabriele, P. M. Comoglio, C. Boccaccio, J. Natl. Cancer Inst. 2011, 103, 645-661.

DOI:10.1093/jnci/djr093

30. R. M. Mohareb, K. M. H. Hilmy, Y. A. Elshehawy, Bull. Chem. Soc. Ethiop. 2018, 32, 285-308. DOI:10.4314/bcse.v32i2.9

31. B. P. McKibben, C. H. Cartwright, A. L. Castelhano, Tetrahedron Lett. 1999, 40, 5471-5474.

DOI:10.1016/S0040-4039(99)01108-9

32. K. Wang, D. Kim, A. Dömling, J. Comb. Chem. 2010, 12, 111118. DOI: $10.1021 /$ cc9001586

33. R. Mishra, K. K. Jha, S. Kumar, I. Tomer, Der Pharma Chem. 2011, 3, 38-54. 


\section{Povzetek}

V prispevku opisujemo serijo heterociklizacijskih reakcij, ki smo jih izvedli na cikloheksan-1,3-dionu s furan-2-karbaldehidom ali tiofen-2-karbaldehidom pri čemer sta nastala ustrezna ilidenska derivata $\mathbf{3 a}, \mathbf{b}$; ti dve spojini sta bili izhodišče za nadaljnje heterociklizacijske reakcije, ki so vodile do tiofenskih in piranskih derivatov 5a-d oz. 6a-d. Ob reakciji spojin 3a,b z elementarnim žveplom in fenil izotiocianatom sta nastala pripojena tiazolska derivata $\mathbf{8 a}, \mathbf{b}$. Pri reakciji s hidrazin hidratom ali fenilhidrazinom pa so nastali 4-hidrazono-4,5,6,7-tetrahidro- $\mathrm{H}$-indazolski derivati 10a-d. Podobno sta pri reakciji med $\mathbf{3 a}$ ali $\mathbf{3 b}$ s hidroksilamin hidrokloridom nastala 6,7-dihidrobenzo[c]izoksazol-4(5H)-on oksima 12a in 12b. Pripravili smo še več drugih heterocikličnih spojin in določili njihove strukture. Pripravljenim spojinam smo določili citotoksične aktivnosti na izbrane celične linije raka. Za najbolj aktivne spojine smo v nadaljevanju določili še inhibitorne lastnosti proti tirozin kinazam in Pim-1 kinazi. 